Déterminants socio-environnementaux des disparités de l'asthme dans l'agglomération de Sfax (centre-est de la Tunisie)

\title{
Mounir Jarraya
}

\section{OpenEdition}

1 Journals

Édition électronique

URL : http://journals.openedition.org/rfst/715

DOI : $10.4000 /$ rfst.715

ISSN : 2492-3672

Éditeur

Espaces et SOciétés (UMR 6590)

\section{Référence électronique}

Mounir Jarraya, « Déterminants socio-environnementaux des disparités de l'asthme dans

l'agglomération de Sfax (centre-est de la Tunisie) », Revue francophone sur la santé et les territoires [En ligne], Miscellanées, mis en ligne le 17 avril 2018, consulté le 06 avril 2021. URL : http:// journals.openedition.org/rfst/715; DOI : https://doi.org/10.4000/rfst.715

Ce document a été généré automatiquement le 6 avril 2021.

La Revue francophone sur la santé et les territoires est mise à disposition selon les termes de la Licence Creative Commons Attribution - Pas d'Utilisation Commerciale - Partage dans les Mêmes Conditions 4.0 International. 


\title{
Déterminants socio- environnementaux des disparités de l'asthme dans l'agglomération de Sfax (centre-est de la Tunisie)
}

\author{
Mounir Jarraya
}

\section{Introduction}

1 Les contrastes sanitaires dans un espace donné font l'objet de plusieurs approches et débats établis par des épidémiologistes, sociologues et géographes... qui tentent de les élucider (Cummins et al., 2007 ; Diez-Roux and Mair, 2010). Deux perspectives peuvent être distinguées. La première (chez les épidémiologistes) vise l'identification des effets des caractéristiques de l'espace sur la santé des individus (Cooper, 2001; White and Borrell, 2011). La deuxième, qui représente l'approche des géographes, cherche à mettre en relation les contrastes sanitaires entre les lieux avec les autres caractéristiques de ces lieux (Rican et al., 2003). L'analyse dans cette approche géographique des inégalités spatiales de santé est centrée sur les entités spatiales en utilisant des échelles variées et parfois emboitées (Riva et al., 2009). A une échelle fine (small areas), Corburn et al. (2006) ont mis en relation les impacts négatifs de certains éléments relevant de l'environnement physique et en particulier l'atmosphère, la pollution du sol et la qualité du logement sur les enfants hospitalisés pour asthme à New York. Cooper (2001) a analysé l'effet de la ségrégation résidentielle à l'intérieur d'une ville sur les inégalités sanitaires entre 47 villes américaines. Il a mis en évidence qu'indépendamment du niveau de revenu moyen dans la ville, des taux de décès plus élevés sont associés à une forte ségrégation résidentielle. Selon une échelle géographique supérieure, Congdon et al. (1997) ont montré des différences de santé entre les "wards" (quartiers) en Angleterre et au Pays de Galles aux profils socioéconomiques similaires mais situés dans des quartiers différents. La prise en compte de plusieurs variables à différents niveaux géographiques a permis d'établir une relation 
entre d'une part, les différences de santé respiratoire entre les 55 plus grandes villes françaises et, d'autre part, les différences de profils socio-économiques et environnementaux de ces villes, ainsi que leur inscription dans différents contextes régionaux et intra-urbains (Aschan-Leygonieet al., 2013).

2 Compte tenu de la multiplicité des déterminants d'une situation sanitaire donnée, les éléments sociaux et environnementaux sont le plus souvent interrogés. Les villes constituent des espaces en perpétuelle évolution et des champs d'étude de la problématique des disparités spatiales sanitaires. Ici, la synergie socioenvironnementale peut fournir des éléments de réponse et d'explication aux thèmes abordés dans de telles problématiques. La notion d'inégalités est fortement utilisée dans ces approches en s'articulant autour de plusieurs thèmes, tels que les inégalités écologiques, les inégalités territoriales, les inégalités face aux risques... (Chaumel et La Branche, 2008). Les inégalités face aux risques par exemple désignent à la fois les inégalités d'exposition et de production du risque sanitaire. Deux notions importantes sont à rapprocher de cette catégorie principale des inégalités écologiques : celle de la « vulnérabilité » (Obrist, 2006) et celle de la « sécurité environnementale » (Lean, 2004). Emelianoff et Theys (2000) insistent sur le fait «qu'il est alors indispensable d'intégrer à la discussion, la notion d'inégalités sociales». Salem et al. (2006) ont montré une relation entre les inégalités spatiales de santé et une accumulation des facteurs défavorables (pauvreté, environnement dégradé, faible accès au soin) et à des comportements sociaux et culturels à risque dans un espace donné.

3 Les approches se sont appuyées sur une maladie ou un ensemble pathologique (pathologies respiratoires, circulatoires, cardiovasculaires...) pour analyser d'abord leur répartition spatiale contrastée et ensuite, l'attribuer à des déterminants de types divers. Parmi ces maladies, figure l'asthme dont 300 millions personnes souffrent à l'échelle mondiale (Baïz et Annessi-Maesano, 2012). En Tunisie, l'asthme touche $6 \%$ de la population générale et en particulier les enfants puisque $10 \%$ de cette classe d'âge seraient asthmatiques (Aissa et al., 2010). L'asthme est une maladie chronique respiratoire, qui fait l'objet de nombreuses recherches sur ses déterminants socioenvironnementaux (Lieutier-Colas et al., 2003 ; Adriel Gudiel et al., 2009 ; Jarraya et Beltrando, 2016). Cette maladie qui se caractérise par des causalités complexes peut représenter un indicateur des inégalités spatiales, dans la mesure où sa morbidité enregistre des niveaux contrastés. Ces inégalités trouvent leurs origines dans des contextes socio-environnementaux différents d'un secteur à l'autre. Plusieurs recherches ont étudié l'asthme du point de vue épidémiologique, en s'intéressant aux facteurs qui déterminent sa variation spatio-temporelle. Laaïdi (1999) a souligné que l'inhalation de l'air froid constitue un facteur de déclenchement de l'asthme intrinsèque, c'est-à-dire de l'asthme lié aux infections bronchiques virales (Busse, 1988). Certains chercheurs ont évoqué le rôle de l'infection virale et le changement de la qualité de l'air intérieur associé à la chaleur pour expliquer cette variation saisonnière (Khot et al., 1984). D'un autre côté, Burnett et al. (1994) ont évoqué l'impact de la pollution de l'air. Ils ont démontré que durant le pic d'ozone et de sulfate observé dans l'Ontario entre mai et août, le changement journalier de la concentration des polluants dans l'air peut être à l'origine d'un accroissement de $7 \%$ d'hospitalisations pour asthme.

4 Dans l'environnement aérien, un grand nombre d'allergènes issus de sources diverses comme le pollen, les acariens, les animaux domestiques... peuvent être à l'origine de 
l'asthme. La part des allergènes dans le déclenchement des symptômes bronchiques comme l'asthme est importante du fait de leur potentielle pénétration plus ou moins profonde dans les voies respiratoires inférieures. Le devenir de ces allergènes inhalés par l'arbre respiratoire est différent selon leur type et leur taille et peut être à l'origine de l'apparition de diverses atteintes bronchiques (Casset et al., 2008).

5 La plupart des allergènes sont soumis aux effets des conditions climatiques. Celles-ci ont un impact capital sur l'environnement, la végétation et sur la dispersion des pollens (Laaïdi et al., 1997 ; Laaïdi, 1999 ; Besancenot, 2001). Ces effets sont perceptibles aussi bien au niveau de la production, de la libération des pollens, lors de la floraison de la plante, qu'au niveau de leur dissémination dans l'espace. Il ressort que la durée d'ensoleillement et la température constituent des vecteurs essentiels de la production et de la dissémination spatiale des pollens et donc de la concentration pollinique de l'air respiré (Thibaudon et al., 2005). Un air fortement concentré par des pollens suffisamment allergisants est à l'origine d'un accroissement des allergies constituant une atteinte sérieuse de l'appareil respiratoire.

6 L'asthme représente une cause importante d'absentéisme aussi bien à l'école qu'au travail, et son coût économique reste très élevé (Deger, 2009). Par exemple, le coût des traitements médicaux et les dépenses sociales de l'asthme était évalué à 7 milliards de francs (1,1 milliard d'euros) en 1994 et il s'est élevé à 1,5 milliard d'euros en France en 2000. L'absentéisme représentait $37 \%$ de ce coût en le considérant comme "coût indirect » de l'asthme (Ministère de l'emploi et de la solidarité, 2001). La communauté des chercheurs s'est ainsi penchée sur les déterminants de la morbidité liée à l'asthme, en privilégiant plutôt les facteurs de risque dits « environnementaux » que les facteurs de risque dits "domestiques» - les conditions d'habitation. L'asthme touche particulièrement les populations urbaines, susceptibles d'habiter à proximité des zones industrielles et d'être exposées à un trafic continu. D'un problème de Santé Publique, l'asthme est alors devenu un enjeu de santé environnementale (Paddeu, 2013. Si ces éléments fournissent de nouvelles pistes pour réfléchir à l'augmentation de l'asthme, la concentration de la prévalence de l'asthme dans les quartiers défavorisés a posé la question du rôle des facteurs socio-économiques, comme le niveau de vie des populations, voire leur origine (Holifield, 2000).

7 L'analyse des déterminants de la morbidité liée à l'asthme constitue un atout pour l'agglomération de Sfax (figure 1). Cette ville localisée sur le littoral Est tunisien et abritant plus de 600000 habitants, est caractérisée par un dynamisme économique important qui lui a permis de devenir le deuxième pôle dans le pays. La plupart des activités (agricoles, industrielles et tertiaires) se développent à Sfax intensifiant ainsi son rôle en tant que métropole régionale rayonnant sur un espace vaste et en particulier sur les gouvernorats de Sud et du Centre-Ouest du pays (Jarraya et Beltrando, 2013). L'installation excessive de l'industrie, matérialisée par la multiplication des zones industrielles au fil du temps dans le Sud comme dans le Nord de la ville de Sfax, est à l'origine d'une pollution intense. Celle-ci a fortement touchée l'environnement et bien évidemment le cadre de vie de la population qui est devenu de plus en plus vulnérable. Une crise environnementale s'instaure par conséquent à Sfax compte tenu ses retombées sur le bien-être et la santé des individus.

8 L'agglomération de Sfax se compose de six délégations qui ont des superficies différentes (figure 1). Depuis l'indépendance, cette ville littorale a vu son infrastructure sanitaire publique se renforcer au fil des décennies avec la mise en place d'un deuxième 
Centre Hospitalo-Universitaire (CHU) (Habib Bourguiba), la création de deux Centres Intermédiaires (structures de soins qui offrent les consultations spécialisées) ainsi que le renforcement continu du réseau des Centres de Santé de Base (CSB) (appelés aussi dispensaires) (figure 2). Les 27 CSB localisés dans l'agglomération de Sfax constituent des structures de soins de première ligne de recours pour la population et offrent les consultations en médecine générale et occasionnellement en pédiatrie. Le CSB dispose généralement d'un médecin et de deux à trois infirmiers. Le nombre des CSB diffèrent d'une délégation à l'autre (figure 2) et nous notons dans ce contexte que la taille de la délégation et l'importance de son arrière pays peuvent justifier leur équipement par ces structures de soins primaires. Il est à rappeler également que tout individu doit s'adresser à un seul CSB, le plus proche du lieu de résidence dans la délégation pour bénéficier de la consultation médicale. Autrement-dit, les patients enregistrés dans un CSB selon les critères appliqués par le secteur de la Santé de Base n'auront pas le droit de consulter ailleurs.

Figure 1 : Description de l'agglomération de Sfax

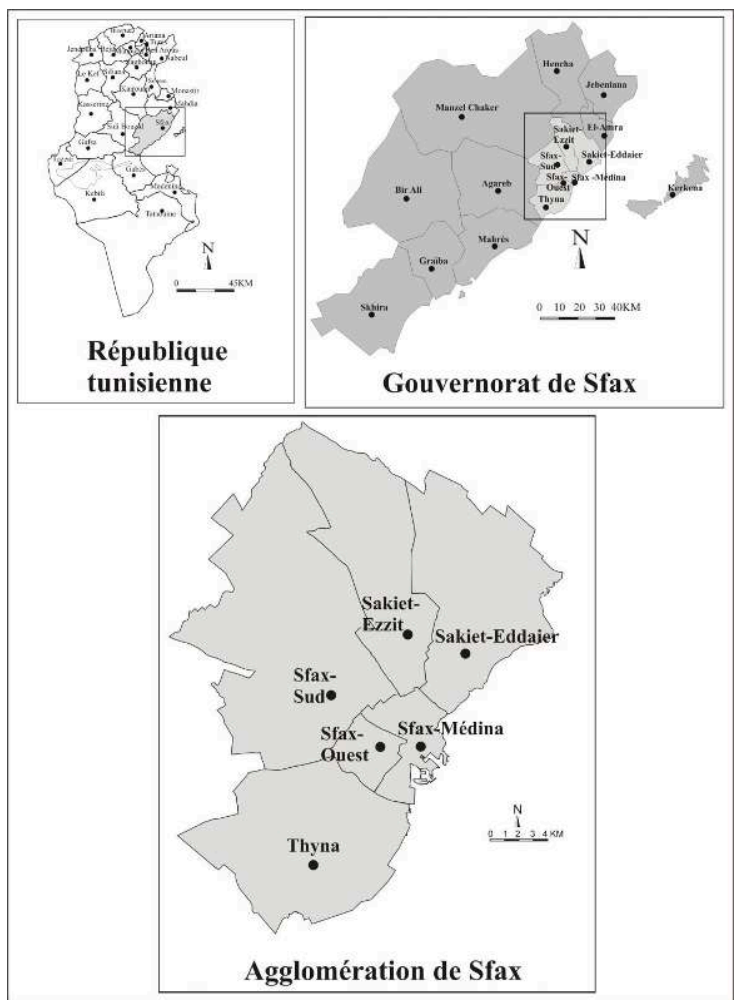

9 Plusieurs considérations commandent notre étude de la morbidité liée à l'asthme dans l'agglomération de Sfax. Cette ville connaît une prévalence de l'asthme (environ $10 \%$ ) plus élevée que la moyenne nationale (6\%) (Aissa et al., 2010; Joobeur et al., 2015) et cette situation est favorisée par de multiples facteurs. Cela est important compte tenu $\mathrm{du}$ rôle économique qu'elle joue à l'échelle nationale et compte tenu du poids démographique, $\mathrm{du}$ contexte social qu'elle représente et de la situation environnementale difficile qu'elle connaît. Nous proposons donc d'analyser les disparités de la morbidité liée à l'asthme dans l'agglomération de Sfax en relation avec certains éléments liés au contexte social et environnemental de la ville. Cette synergie sanitaire-sociale-environnementale implique la recherche des mesures d'intervention 
afin d'améliorer le bien-être et la santé d'une population qui souffre depuis des décennies.

Figure 2 : La répartition des structures de soins publiques dans l'agglomération de Sfax

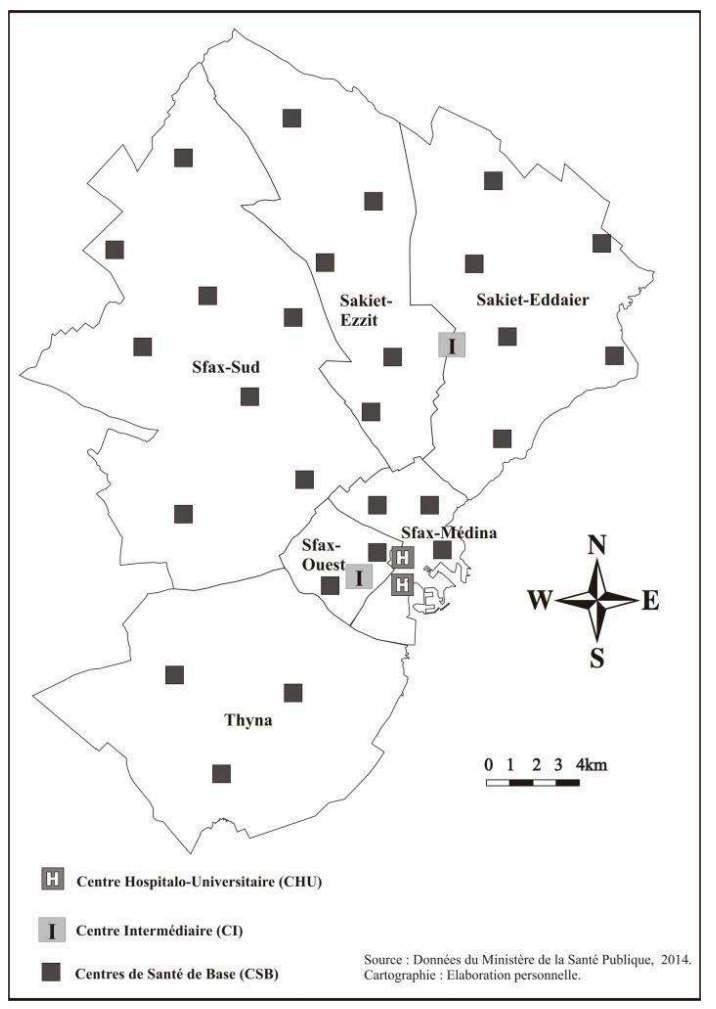

\section{Données et méthodes}

10 L'analyse du contexte socio-environnemental de la morbidité de l'asthme dans le Grand Sfax a nécessité l'utilisation d'informations issues de diverses sources couvrant la période 2009-2016. Les données de consultations pour asthme ont été fournies par la Direction Régionale de la Santé Publique à Sfax. Les données démographiques ont été obtenues auprès de l'Institut National de la Statistique (INS). En l'absence d'informations relatives au contexte de vie des patients (lieu de résidence, conditions de logement, emploi, état tabagique...) et à la maladie (ancienneté, symptômes, fréquence de crises, types d'allergènes, types de traitement...), nous avons mené une enquête de 10 jours dans dix Centres de Santé de Base (CSB) ( 1 jour dans chaque CSB), en avril (06-16 avril) et en octobre (05-16 octobre) 2015 (figure 3). Le nombre total des patients enquêtés est de 187 durant les deux périodes et se répartissent différemment entre les CSB (figure 4). Nous avons mené des entretiens avec les patients, les médecins en poste, qui ont rempli une fiche d'enquête (voir annexe), et les pharmaciens. D'autres entretiens ont été effectués également avec les pharmaciens des officines privées dans la ville de Sfax. D'autres entretiens qualitatifs ont été conduits avec les patients afin de collecter certaines informations indispensables comme celles relatives à leurs conditions de logement et à la question du tabagisme. La période du déroulement de l'enquête a été choisie en avril et en octobre, puisque la morbidité d'asthme enregistre deux pics pendant ces deux mois. Notre choix de ces dix CSB est justifié par le fait qu'ils se répartissent dans les différents secteurs du tissu urbain de l'agglomération de Sfax et 
drainent des espaces occupés différemment par la population et présentant des facteurs de risque différents et potentiellement capitaux pour l'asthme. D'autres éléments liés d'une part à notre capacité d'exécuter et de gérer un tel travail d'investigation, et d'autre part, aux conditions d'accueil dans les CSB et à la coopération des médecins parfois incertaine, a orienté notre choix de ces CSB. L'importance majeure de ces enquêtes réside dans le fait qu'elles nous permettront de déceler les déterminants potentiels de l'asthme afin d'expliquer un niveau de morbidité donné dans tel secteur de l'agglomération Sfax.

11 Concernant la méthodologie suivie dans le présent travail, nous chercherons tout d'abord à montrer comment la morbidité d'asthme est exprimée par les consultations médicales enregistrées dans les Centres de Santé de Base de l'agglomération de Sfax. Ensuite, nous tenterons d'analyser la part des déterminants socio-environnementaux, que nous avons classés en facteurs intrinsèques et facteurs généraux, dans l'explication des disparités spatiales de l'asthme. Cette catégorisation de déterminants est justifiée par le fait que l'ensemble de la population de l'agglomération est soumis aux effets des phénomènes (comme le vent et les pollens) de grande ampleur spatiale impliquant que personne n'est à l'abri de leurs répercussions. C'est pour cela que nous les avons qualifiés de "facteurs généraux ». A l'inverse, d'autres facteurs liés au comportement humain (tabagisme), aux conditions de vie et à la configuration environnementale de l'espace occupé (pollution atmosphérique générée par l'activité industrielle) sont qualifiés «facteurs intrinsèques». Ces facteurs, qui intéressent l'enveloppe socioenvironnementale spécifique à chaque délégation (et donc ils diffèrent d'une délégation à une autre), nous permettent de suggérer qu'elles déterminent la variation spatiale de la morbidité liée à l'asthme. Enfin, nous essayerons d'analyser les disparités spatiales de la morbidité liée à l'asthme à l'échelle de l'agglomération en calculant les moyennes (de consultations par délégation) et les indicateurs sanitaires (nombre moyen de consultations par CSB), en nous basant sur la cartographie des variables exprimant la morbidité (les consultations médicales) et sur les résultats des enquêtes conduites en avril et octobre 2015, afin de diagnostiquer le paysage épidémiologique saisonnier de l'asthme et d'expliquer sa variation spatio-temporelle. 
Figure 3 : Le réseau de déroulement de l'enquête sanitaire en avril et octobre 2015

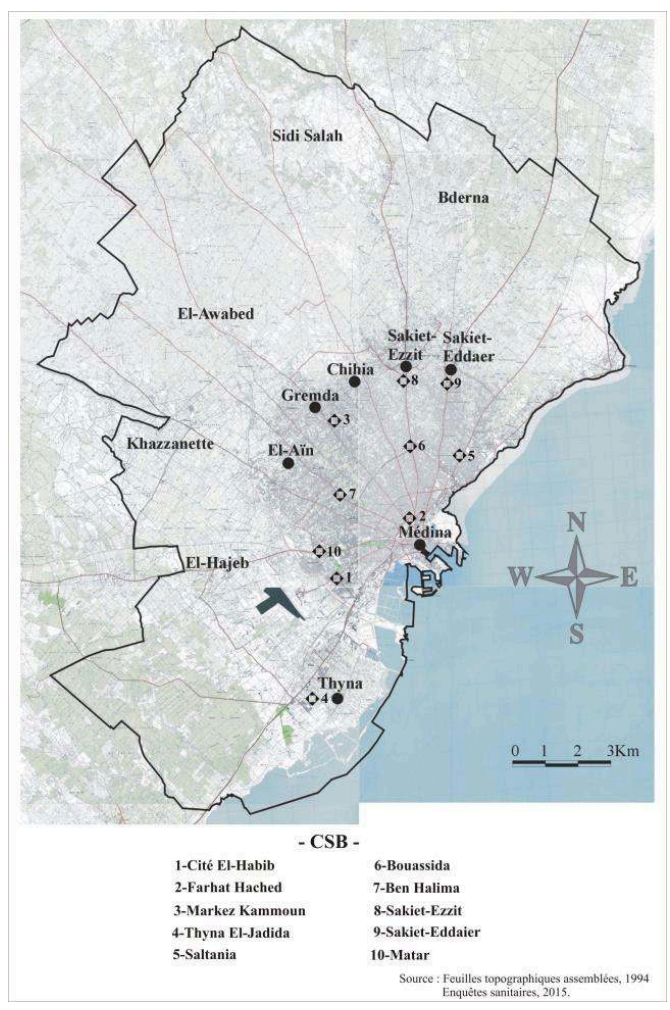

Figure 4 : La répartition de l'effectif des patients enquêtés selon les CSB en avril et octobre 2015

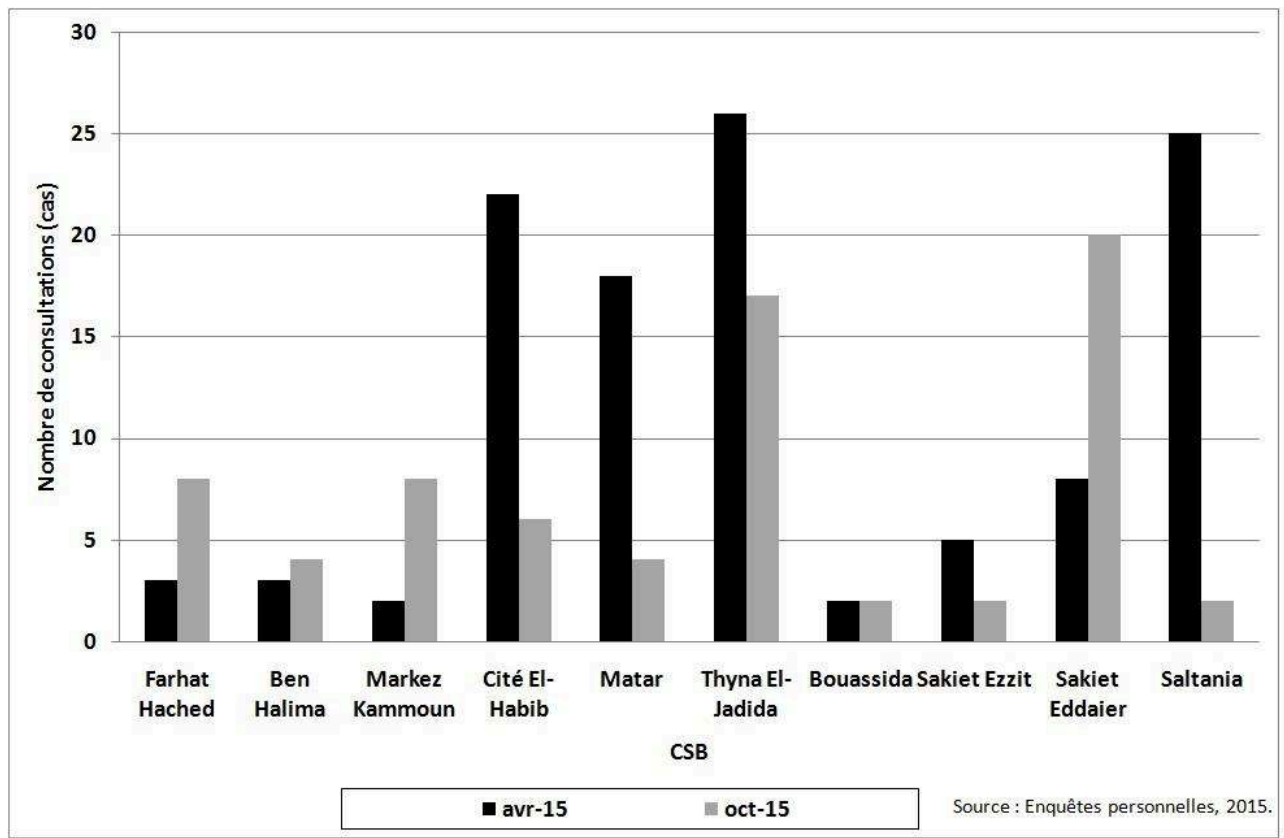




\section{Epidémiologie de l'asthme dans l'agglomération de Sfax}

12 L'asthme est une maladie respiratoire, qui constitue un motif fréquent des consultations médicales dans le secteur de la Santé de Base dans le Grand Sfax et dont l'analyse nécessite la connaissance de sa typologie c'est-à-dire la catégorie des pathologies auxquelles il appartient, et la variation de sa morbidité selon l'âge au niveau des personnes atteintes. L'étude de la variation temporelle des consultations pour asthme permet également de mettre en évidence le régime saisonnier de sa morbidité à Sfax.

\section{Le poids de l'asthme dans sa catégorie pathologique}

13 La part de l'asthme dans la catégorie des maladies chroniques est de $4,8 \%$, sur la période 2009-2016, dans le Grand Sfax. Cette catégorie regroupe des pathologies ayant des prévalences élevées comme l'hypertension artérielle (HTA), le diabète,... Bien que relativement faible, la part de l'asthme montre qu'une partie de la population souffre d'une maladie respiratoire qui altère son bien-être et sa santé et peut mettre sa vie en jeu.

\section{Une morbidité contrastée selon l'âge}

14 Les données de consultations pour l'asthme sont enregistrées selon quatre classes d'âge : les enfants ( $\leq 5$ ans et 6-14 ans), les jeunes (15-24 ans) et les adultes plus âgés ( $\geq$ 25 ans). L'effectif annuel moyen (3623 cas) enregistré sur la période 2009-2016 se répartit inégalement entre les catégories d'âge. En effet, la part des catégories d'âge dans l'effectif total des cas d'asthme varie de 3,4 \% au niveau de la catégorie (6-14 ans) à $84,2 \%$ au niveau de la catégorie ( $\geq 25$ ans) (figure 5 ). Nous déduisons que l'asthme touche beaucoup plus la catégorie des adultes puisqu'avec l'avancement d'âge, l'individu devient de plus en plus exposé et sensible aux agents multiples qui altèrent son bien-être et son état de santé respiratoire (Besancenot, 2001). Donc, c'est le cumul de l'exposition durant les années de leur vie qui justifie la part importante des adultes dans l'effectif des consultations pour asthme (Devouassoux, 2003; Thomson, 2013). Cependant, nous signalons que nous avons adopté la classification des patients selon les catégories d'âges utilisées par la Direction Régionale de la Santé Publique de Sfax. Une telle classification présente plusieurs points de faiblesse telles que l'étendue de la dernière classe ( $\geq 25$ ans) qui met les personnes jeunes et âgées dans la même catégorie. Nous n'avons pas pu résoudre ce problème car les données de base sont enregistrées selon cette classification. 
Figure 5 : Distribution des consultations pour asthme par classe d'âge dans le Grand Sfax (2009-2016)

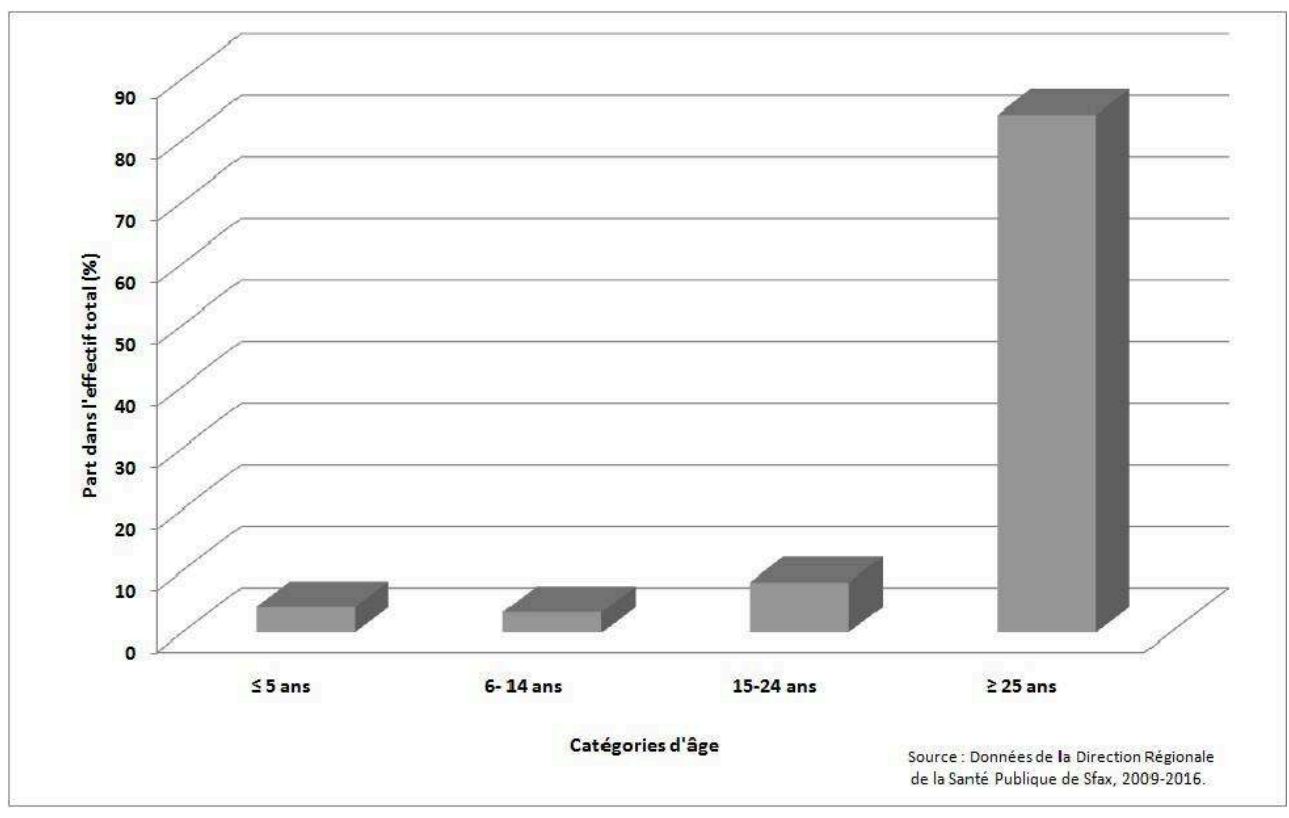

\section{Quelques aspects de saisonnalité de la morbidité liée à l'asthme}

L'étude de la répartition des consultations pour asthme en fonction du temps dans le Grand Sfax indique un régime saisonnier caractérisé par deux pics (figure 6). Le premier pic, qui est le plus important, survient au printemps puisque les consultations pour asthme atteignent 447 cas en avril.

Figure 6 : Variation mensuelle moyenne des consultations pour asthme dans le Grand Sfax (2009-2016)

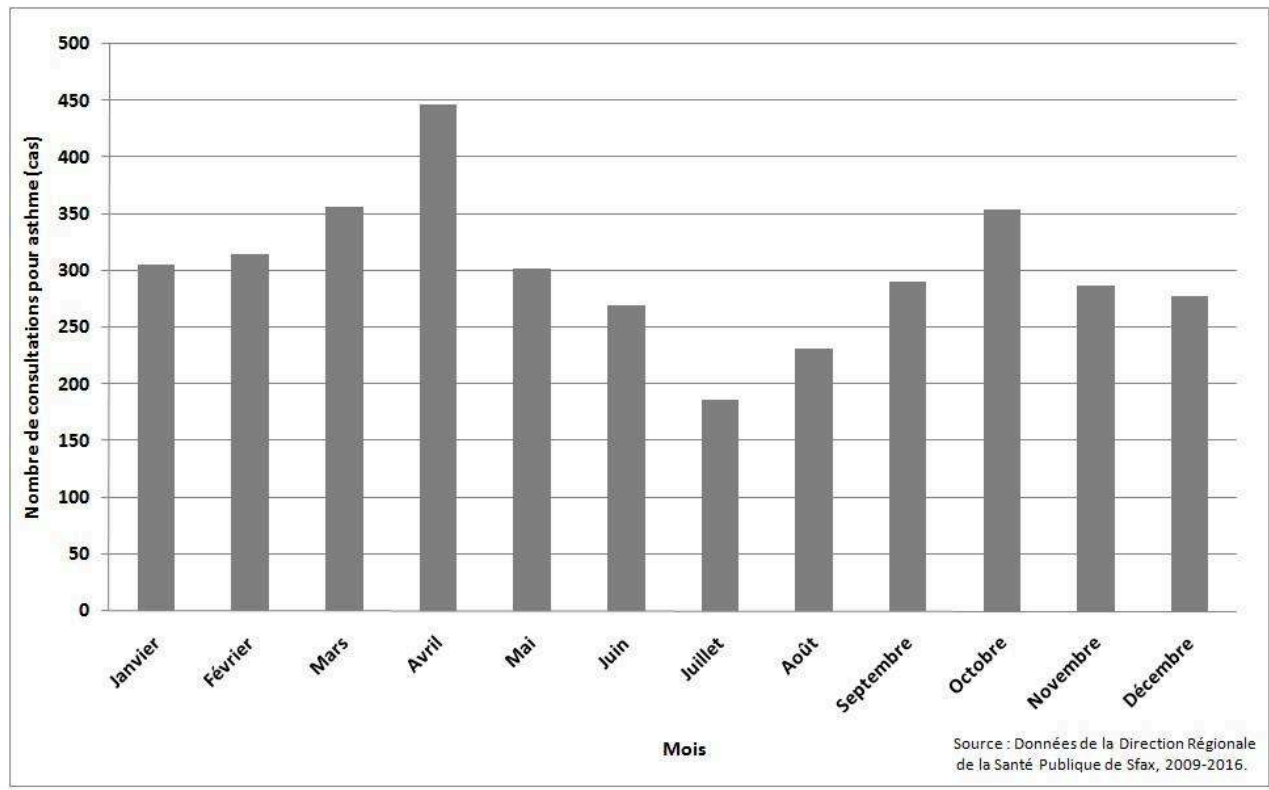

16 Le deuxième pic survient en automne (octobre), dans la mesure où les CSB enregistrent 354 consultations pour asthme. Cette variation saisonnière des consultations pour 
asthme suscite la recherche des éléments qui l'expliquent. Il est connu que l'asthme est une maladie chronique persistante chez les patients (Turki, 2008), ce qui explique la permanence des consultations pour cette maladie dans le secteur de la Santé de Base tout au long de l'année. Cependant, les deux pics printanier et automnal montrent qu'un autre facteur s'ajoute pour expliquer la hausse des consultations pour asthme. C'est à l'environnement extérieur qu'il faut attribuer cet effet temporaire, et plus précisément aux allergènes végétaux au printemps et à la poussière de sable en automne. Nous analyserons cet aspect dans la partie suivante.

17 Outre la situation épidémiologique de la morbidité de l'asthme et sa variation temporelle, l'analyse de la répartition spatiale des consultations dans l'agglomération de Sfax dévoile une liaison avec le contexte socio-environnemental.

\section{La spatialisation de la morbidité de l'asthme : le reflet des disparités socio-environnementales}

18 La répartition spatiale des consultations pour asthme enregistrées par les CSB selon les délégations du Grand Sfax est soumise à de multiples déterminants d'origines diverses.

\section{La variation spatiale de la morbidité d'asthme}

19 La spatialisation du nombre moyen de consultations pour asthme enregistrées par délégation sur la période 2009-2016 montre des disparités, particulièrement entre SfaxMédina ( 469 cas) et Sfax-Sud ( 775 cas) (figure 7). Cette répartition différenciée de la morbidité liée à l'asthme est déterminée par des facteurs intrinsèques à chaque délégation et à des facteurs généraux « plus synoptiques » qui intéressent la totalité de l'espace de l'agglomération de Sfax. 
Figure 7 : Variation spatiale des consultations pour asthme dans le Grand Sfax (2009-2016)

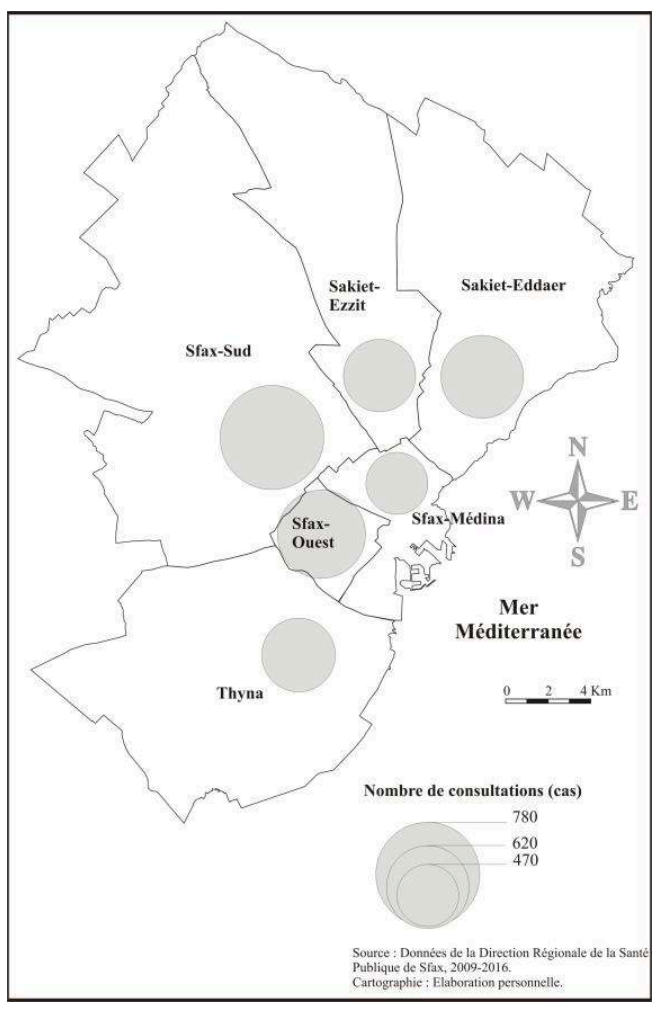

\section{Les éléments intrinsèques} expliquer les disparités spatiales observées au niveau de la morbidité de l'asthme dans l'agglomération de Sfax.

\section{La taille de la population}

La taille démographique des délégations constitue un facteur qui détermine les demandes en matière de soins par les habitants. Théoriquement, les espaces les plus peuplés enregistrent le volume de consultations le plus élevé. En effet, la population des délégations de l'agglomération de Sfax varie de 119139 habitants à Sfax-Sud à 62997 à Thyna (figure 8). Nous observons que la délégation de Sfax-Sud, qui se caractérise par sa taille démographique importante (119139 habitants) enregistre l'effectif de consultations le plus élevé (775 cas) (figure 7), alors que la délégation de Thyna, qui abrite l'effectif d'habitants le plus bas dans l'agglomération (62 997 habitants), enregistre un volume faible de consultations (552 cas) (figure 7). Thyna est une délégation moins peuplée mais elle connaît depuis une décennie une urbanisation croissante et cela explique le niveau de consultations assez faible. Cependant, bien qu'elle soit importante, la taille démographique à Sfax-Médina est contrariée par un niveau bas de consultations pour asthme. Cela est expliqué par les mutations fonctionnelles connues depuis des décennies par le centre-ville qui a perdu sa fonction résidentielle contre un développement des activités tertiaires.

Certes, la taille démographique détermine l'importance des consultations pour asthme dans les CSB. Nous nous rappelons à ce propos que selon la réglementation, chaque 
habitant s'adresse obligatoirement au CSB le plus proche de son lieu de résidence dans sa délégation, autrement dit il n'a pas le droit de consulter dans les structures de soins situées dans les autres délégations (Jarraya, 2009). Cependant, ce facteur démographique n'est pas le seul. D'autres éléments interviennent pour expliquer les disparités spatiales de la morbidité liée à l'asthme entre les délégations.

Figure 8 : Répartition de la taille de la population par délégation en 2014

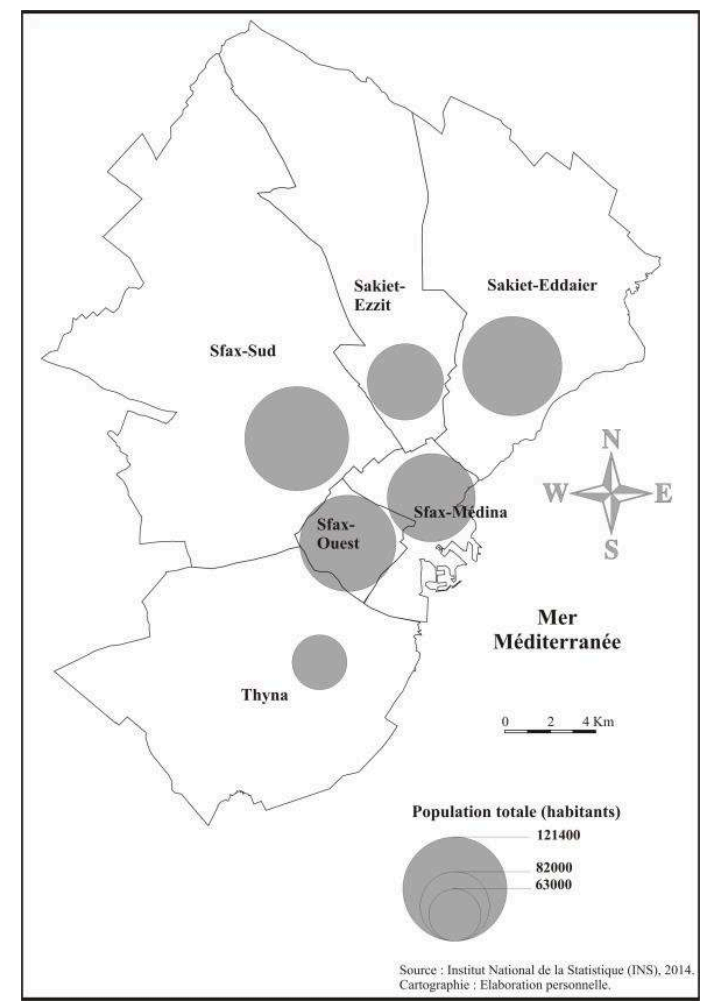

\section{Les conditions d'habitat : un indicateur du niveau de vie}

Les conditions d'habitat et l'état du logement constituent un révélateur pertinent du niveau de vie des populations. Leurs répercussions sur la santé sont multiples et se manifestent différemment. Nos enquêtes effectuées au niveau de dix CSB (figure 3) en avril et en octobre 2015, sur 187 asthmatiques consultants, montrent que $67 \%$ des cas vivent dans des milieux qui peuvent favoriser les atteintes du système respiratoire, en particulier l'asthme. En effet, ces individus habitent dans des logements peu ventilés et peu exposés au rayonnement solaire. Parmi les causes d'une affection asthmatique, nous citons l'humidité accrue qui est favorable à la prolifération des acariens. L'humidité permanente des pièces et la faible ventilation affecte la qualité de l'air, et constitue une condition propice à l'apparition d'une allergie aux acariens.

Il ressort de nos entretiens avec des malades asthmatiques résidant dans les quartiers populaires (cités El-Bahri 1, El-Habib...) polarisés par le CSB Cité El-Habib (28 patients asthmatiques), qui se caractérisent par des habitations très serrées, que certaines pièces de leur logement sont dépourvues de ventilation et que l'humidité constitue une gêne permanente pour leur bien-être. Ils ajoutent qu'après ils sont devenu(e)s asthmatiques et que les acariens en constituent la cause principale. Ces mêmes malades ont affirmé lors de nos entretiens que leur état risque de s'aggraver dans le futur s'ils 
n'améliorent pas les conditions de leur logement. Cependant, il faut se rappeler que les habitants des cités populaires appartiennent à la classe sociale la moins favorisée dont les moyens sont très limités surtout pour assurer le traitement de l'asthme, car les médicaments sont chers et dépassent leurs moyens.

Dans le même contexte, nos entretiens effectués avec 27 patients asthmatiques au CSB Saltania (figure 3), qui draine une population occupant des espaces humides laissent apparaître un effet capital de l'humidité. Celle-ci est due à deux facteurs : d'abord, la proximité à la mer qui rend l'air humide en permanence et affecte directement les logements. En outre, les habitants de ce secteur déplorent la montée des eaux de la nappe phréatique qui a fortement dégradé leurs maisons. Ce phénomène aggrave de plus en plus le problème épineux de l'humidité et affecte la qualité de vie dans les logements. Le bien-être se trouve ici dégradé car les sources de gêne sont multiples. La promiscuité des logements serrés dans la cité Bourguiba, qui sont déjà peu ou mal ventilés et peu exposés au soleil, ainsi que la proximité à la mer accentuent l'humidité et par conséquent offrent les conditions propices pour que les acariens prolifèrent ce qui accroit le risque d'asthme.

Généralement, un habitat sain, bien ventilé et ensoleillé constitue une condition primordiale pour lutter contre l'asthme provoqué par l'allergie aux acariens (Devouassoux, 2003).

27 Cette condition est fortement liée au niveau de vie puisque les classes sociales défavorisées occupent généralement des espaces qui ne respectent pas les normes demandées pour un cadre de vie sain, tels que les espaces limitrophes aux zones industrielles.

\section{La proximité des zones industrielles}

Sfax est une ville industrielle importante à l'échelle nationale. Les industries se localisent dans les zones littorales au Nord (Poudrière 1 et 2) et du Sud de la ville (Oued El-Maou, Chaabouni, Thyna). Par leurs rejets liquides et solides, ces unités industrielles ne cessent de dégrader la qualité de l'air et de l'eau continentale et marine (Choura, 2008). Sous l'effet de la densification urbaine galopante, plusieurs quartiers (en particulier populaires) se trouvent à proximité des zones industrielles (figure 9). Source : Dahech et Bouaziz, 2012 
Figure 9. Proximité des quartiers résidentiels aux zones industrielles au Sud de la ville de Sfax

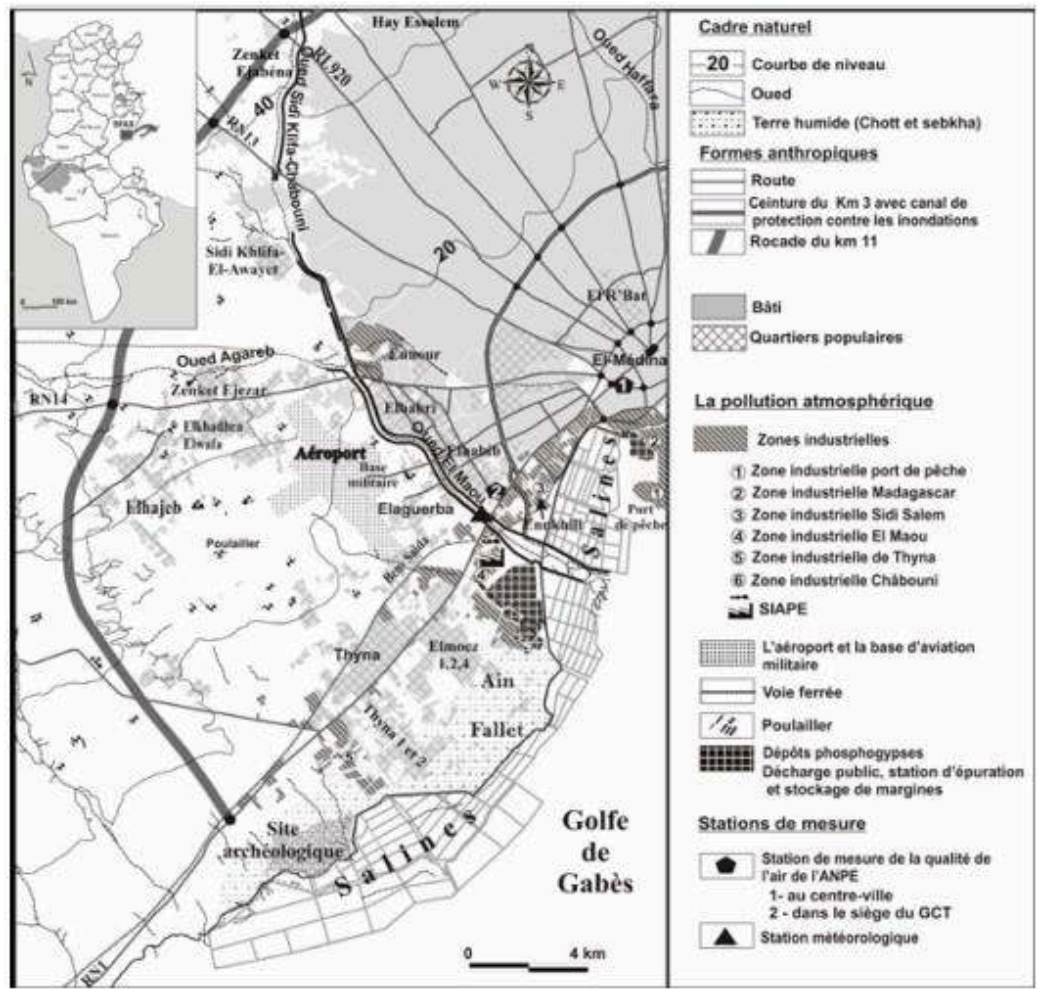

Pour montrer le rôle de la proximité aux zones industrielles dans l'explication des niveaux élevés de morbidité d'asthme, nous avons utilisé l'indicateur du nombre moyen de consultations / CSB. La spatialisation de cet indicateur montre que les délégations de Sfax-Ouest et Thyna arrivent en tête avec respectivement 221 et 124 cas (figure 10). Les CSB de ces deux délégations drainent une population résidant dans des cités populaires (Cités El-Bahri 1, El-Bahri 2, El-habib, El-Moez...) qui connaissent une densification croissante au fil du temps ainsi que des conditions insatisfaisantes d'habitat pour le bien-être et la santé des individus. Leur proximité aux zones industrielles constitue un facteur capital pour expliquer la morbidité élevée de l'asthme. 
Figure 10 : Variation spatiale du nombre moyen de consultations par CSB (2009-2016)

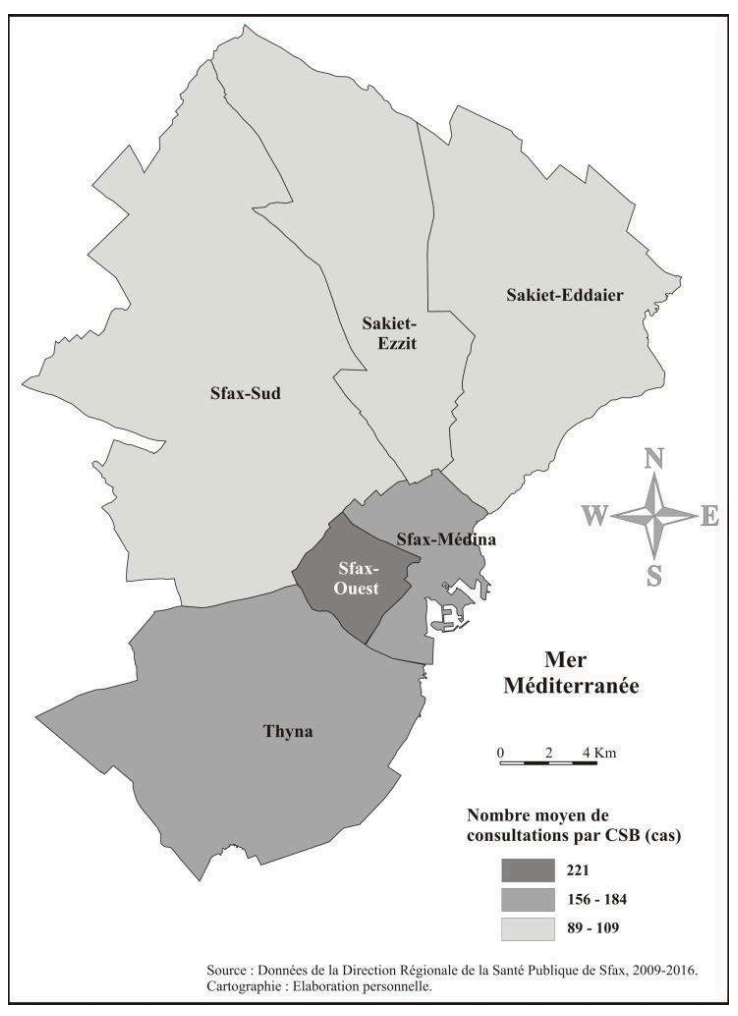

Pour analyser l'éventuel effet de la proximité des zones industrielles dans la morbidité élevée liée à l'asthme, nous nous sommes focalisés dans nos enquêtes et nos entretiens sur les secteurs polarisés par les CSB de Matar, Cité El-habib (pour la délégation de Sfax-Ouest) et le CSB de Thyna El-Jadida (pour la délégation de Thyna). Il ressort de nos investigations que les émissions gazeuses rejetées dans l'atmosphère par les unités industrielles environnantes pourraient contribuer à la fragilisation de la santé des personnes. Outre la dégradation du cadre de vie (mauvaises odeurs, air impur, visibilité insuffisante...), les répercussions sur la santé respiratoire sont multiples. Par exemple, parmi les 28 asthmatiques qui ont consulté le CSB de Cité El-Habib en avril et octobre 2015, 80 \% (22 patients) déclarent que leur malaise commence à apparaître quand l'air respiré devient trop chargé par les polluants. Un vent soufflant de l'Est ramène les polluants vers ces cités résidentielles environnantes et contribue à une forte concentration de l'air. Les symptômes indiquant une réactivation d'une allergie respiratoire varient de signes banals (picotement des yeux, inflammation du nez et éternuement...) à d'autres plus sérieux comme la toux, les difficultés de respiration et le déclenchement des crises d'asthme pour les personnes hypersensibles. Cette situation est expliquée par l'installation des industries chimiques dans ce secteur méridional de la ville auquel sont attribués les niveaux de pollution les plus élevés.

31 L'industrie chimique vise le traitement des phosphates. La première unité, la Société Industrielle d'Acide Phosphorique et d'Engrais (SIAPE), a été implantée en 1952 suffisamment loin de la population.

Elle se trouve actuellement en plein centre de la commune de Thyna du fait de l'expansion urbaine. Cette usine s'étale sur 130 hectares. La SIAPE est la plus polluante des industries de la ville (Azri et al., 2000). Ses émissions gazeuses permanentes sont dégagées dans l'atmosphère par l'intermédiaire de grandes cheminées (figure 11). Les 
volumes de gaz dégagé annuellement dans l'atmosphère sont essentiellement chargés en oxyde de soufre (SO2) (13472 tonnes/an), en composés fluorés (1352 tonnes/an) et en poussières (2266 tonnes/an). A titre d'exemple, la quantité de fluor rejetée par la SIAPE représente $95 \%$ de l'émission totale de ce gaz à Sfax (Dahech, 2007). Leur dispersion est fortement influencée par les conditions météorologiques, en particulier le vent et les inversions thermiques journalières qui peuvent transporter les polluants loin de leurs sources d'émission ou les accumuler dans le milieu environnant.

Figure 11 : Les émissions gazeuses par la SIAPE

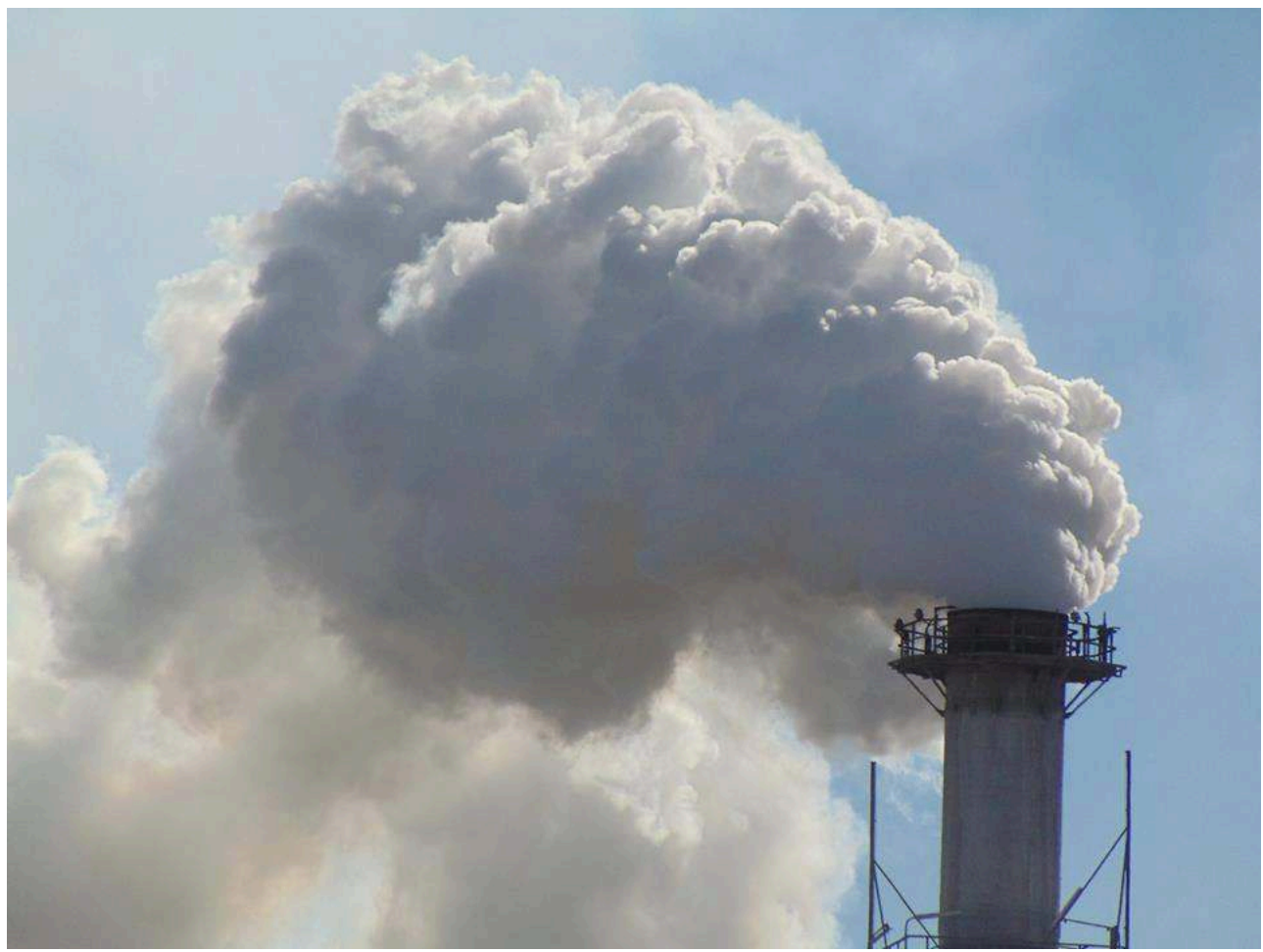

Source : http://highlights.com.tn/sfax-face-a-la-determination-des-habitants-la-siape-compte-sesheures/

Nous avançons ici les résultats des travaux qui ont traité la question de la pollution atmosphérique générée par la SIAPE. Selon Kallel (2008), la valeur moyenne de SO2 enregistrée à l'échelle horaire en juin $(6843 \mu \mathrm{g} / \mathrm{m} 3)$ et juillet $2006(2646 \mu \mathrm{g} / \mathrm{m} 3)$ reflète des dépassements importants de la valeur limite recommandée par l'Organisation Mondiale de la Santé (OMS) ( $350 \mu \mathrm{g} / \mathrm{m} 3)$.

Dans l'étude menée par Dahech et al. (2006), il a été démontré que les normes européennes pour le SO2 (2008/50/CE), qui préconisent une moyenne horaire de $130 \mathrm{ppb}$ à ne pas dépasser plus que 24 fois durant l'année, n'ont pas été respectées puisque 350 dépassements sont enregistrés au niveau de la station de mesure installée au siège du Groupe Chimique Tunisien (CGT) dans la zone industrielle. De plus, des niveaux très élevés de SO2 ont été enregistrés, comme le 6 juin 2006 à $3 \mathrm{~h}$ (2563 ppb), 7h $(2131 \mathrm{ppb})$ et à $23 \mathrm{~h}(2152 \mathrm{ppb})$. Concernant le PM10 (particules dont le diamètre est inférieur à 10 micromètres), la moyenne annuelle horaire de l'émission de PM10 est de l'ordre de $91,7 \mu \mathrm{g} / \mathrm{m} 3$, alors que la valeur limite de cette moyenne pour sauvegarder la santé des individus est de $40 \mu \mathrm{g} / \mathrm{m} 3$ selon la réglementation française du 10 mars 2011, en matière de qualité de l'air (Dahech et al., 2006). 
être et l'apparition des signes morbides sont associées aux situations de calme anticyclonique marqué par l'absence d'un vent actif. Ces situations qui sont généralement associées des brises thermiques (de mer et de terre) se caractérisent par une vitesse de vent faible voire nulle, ce qui affaiblit la dispersion verticale et horizontale des polluants. Cela aboutit à une concentration maximale de l'air par les gaz et les poussières émis par la SIAPE ainsi qu'à leur dépôt dans les secteurs limitrophes densément peuplés (figure 12).

Figure 12 : Le site de la SIAPE et ses environs urbains

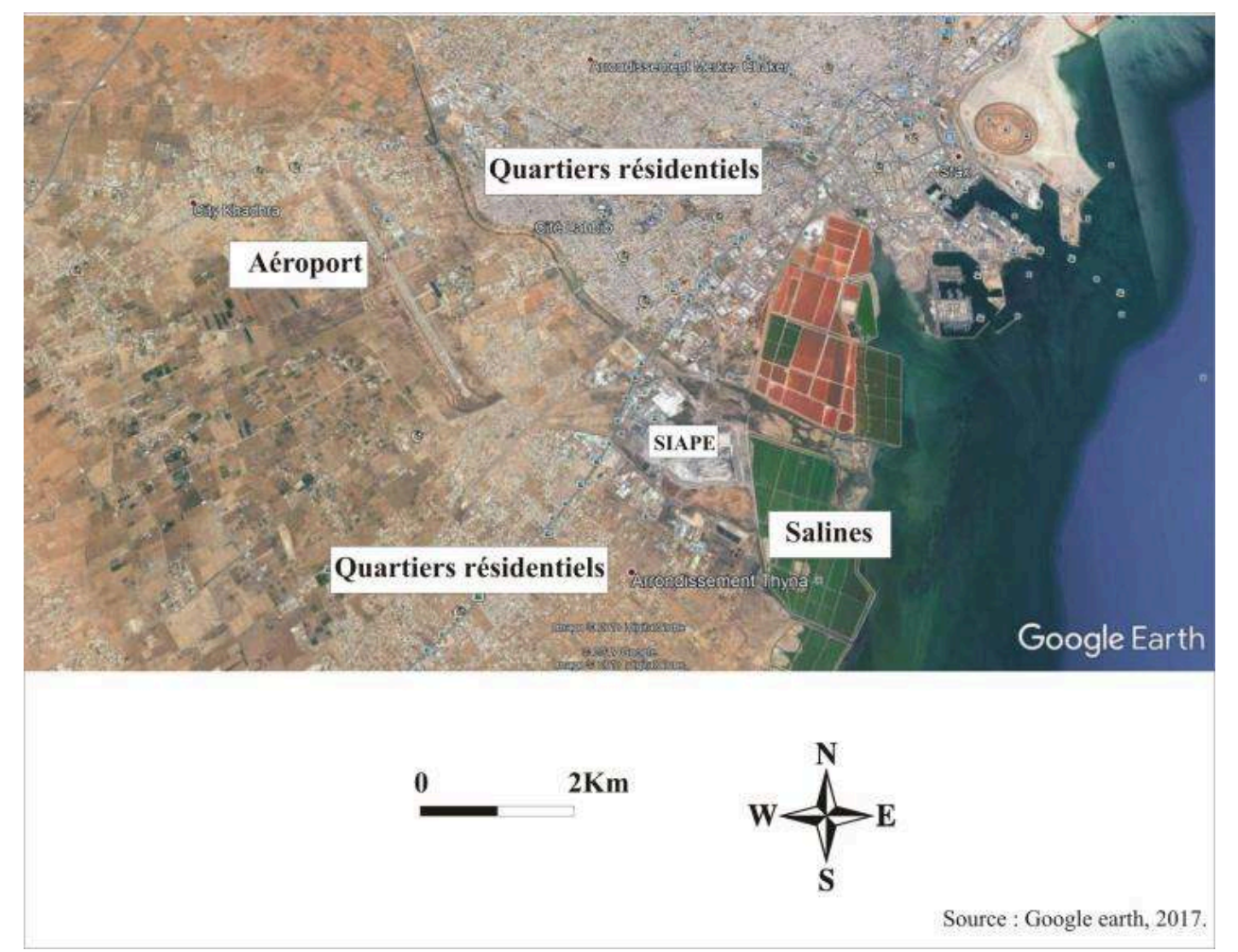

Nos investigations dans le CSB de Thyna El-Jadida, montrent que $63 \%$ des asthmatiques interrogés (43 patients) confirment leur sensibilité aigue à la dégradation de la qualité de l'air respiré provoquée par les émissions des unités industrielles, en particulier la SIAPE. Des sensations de suffocation et de détresse respiratoire aigue surviennent suite aux situations de calme. La faiblesse du vent empêche la diffusion des polluants loin du site d'émission dans les deux sens, vertical et horizontal. Cela aboutit à une forte 
concentration de l'air respiré en polluants et donc la dégradation de sa qualité. Ils ont ajouté aussi que la situation devient critique en été et en automne puisque la forte concentration des gaz polluants est associée à la forte chaleur, à l'humidité élevée et à la faible vitesse de vent. Cela favorise le déclenchement des crises d'asthme ( $15 \%$ des cas) plus ou moins aigues pour les personnes très vulnérables, ce qui a imposé à plusieurs reprises leur admission dans le service d'urgence du CHU Habib Bourguiba pour se mettre sous l'oxygène.

\section{Le facteur de tabagisme}

38 Ce facteur socio-comportemental constitue l'un des déterminants de la santé des asthmatiques. S'il est difficile de savoir si le tabagisme actif peut amener à l'apparition de l'asthme chez une personne qui n'en avait pas, il est certain que ce facteur aggrave la maladie et altère la santé respiratoire (Thomson et al., 2013). Il ressort de nos enquêtes établies en 2015 que 80 \% des hommes asthmatiques ( 84 hommes) sont des tabagiques actifs. Ces personnes affirment que leurs crises d'asthme sont plus sévères et que l'efficacité de leur traitement est réduite par rapport aux malades non tabagiques. Nous avons interrogé aussi les médecins traitant dans les CSB, qui ont souligné que la fumée du tabac renferme entre autres substances nocives, des irritants puissants. Ceux-ci ont tendance à accentuer la fermeture des bronches, car l'irritation qu'ils provoquent augmente l'inflammation et la production de secrétions. En outre, ils ont affirmé que la cortisone à inhaler, traitement de base de l'asthme, est moins efficace chez le fumeur.

39 Du côté des tabagiques passifs, les femmes asthmatiques dont les maris sont fumeurs constituent la première catégorie vulnérable. Ainsi, $85 \%$ des femmes asthmatiques enquêtées (103 femmes) ont exprimé leur gêne voire leur souffrance de l'effet du tabac, tout en soulignant que leur état de santé se dégrade de plus en plus et que le traitement antiasthmatique devient de moins en moins efficace. De plus, elles ont affirmé que le tabagisme est devenu une source de désaccord avec leurs maris incompréhensifs. En outre, les enfants font aussi partie de cette vulnérabilité accrue au tabagisme. Nous avons signalé ci-dessus que les enfants représentent ensemble 7,6 \% (soit 14 enfants) de l'effectif total des consultations pour asthme. Nos investigations montrent que pour la quasi-totalité d'entre eux ( $95 \%$ des cas), la présence d'un père tabagique fragilise davantage leur état de santé respiratoire puisque l'asthme devient de plus en plus aigu. Les médecins en poste confirment que l'aggravation de l'asthme de l'enfant par le tabagisme passif est incontestable et que l'enfant asthmatique dont l'entourage familial fume a des crises plus fréquentes, des capacités respiratoires plus basses et des besoins en médicaments antiasthmatiques plus importants que si ses proches ne fument pas.

40 Tandis que des éléments intrinsèques de la variation de la morbidité pour asthme soulignent les spécificités de chaque délégation, mettant ainsi en évidence un effet spatial local, d'autres éléments plus généraux exposent la totalité de l'agglomération de Sfax aux risques environnementaux : c'est le cas de la poussière et des pollens.

\section{Les éléments généraux}

41 Nous avons choisi de qualifier ces éléments de "généraux ", car les phénomènes qui les génèrent sont synoptiques et touchent, avec des degrés cependant variables, l'ensemble des habitants de l'agglomération de Sfax. 


\section{L'effet de la poussière liée au vent de sable}

\section{l'air (figure 13A).}

Figure 13A : Le ciel voilé associé à un vent de sable à Sfax en octobre 2015

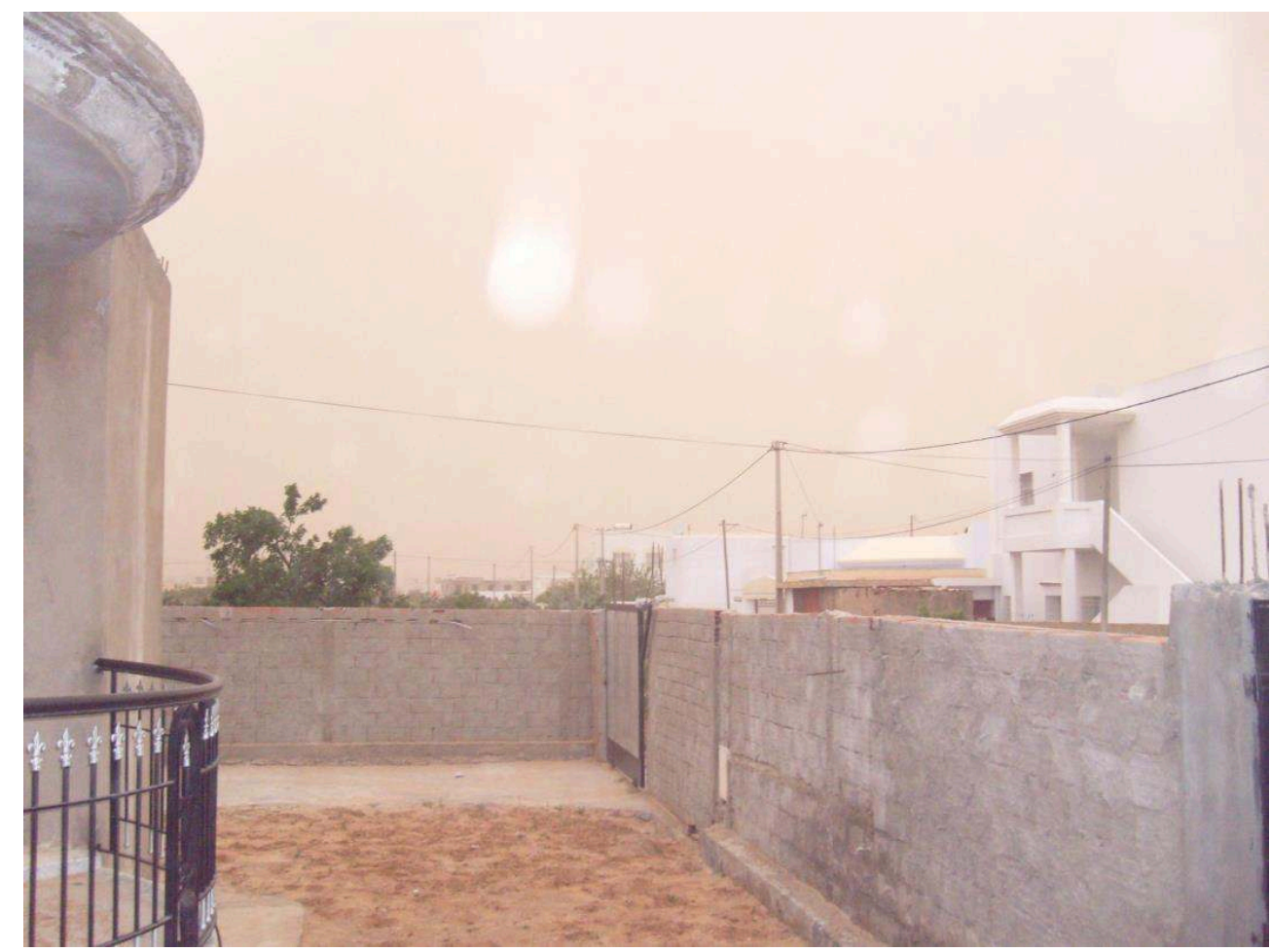

Source : Clichés Jarraya M., 2015.

Ce type de temps est inconfortable. En outre, le fait qu'il soit peu fréquent, augmente le degré de vulnérabilité humaine en raison de l'absence de dispositif d'adaptation ou de lutte contre ce phénomène (Dahech et Beltrando, 2012). Le vent de sable a des effets nocifs sur l'environnement (accumulation de sable, érosion,...) (figure 13B) et sur la santé, notamment pour les personnes vulnérables. 
Figure 13B : Accumulation de sable fin sur la véranda d'une maison en octobre 2015

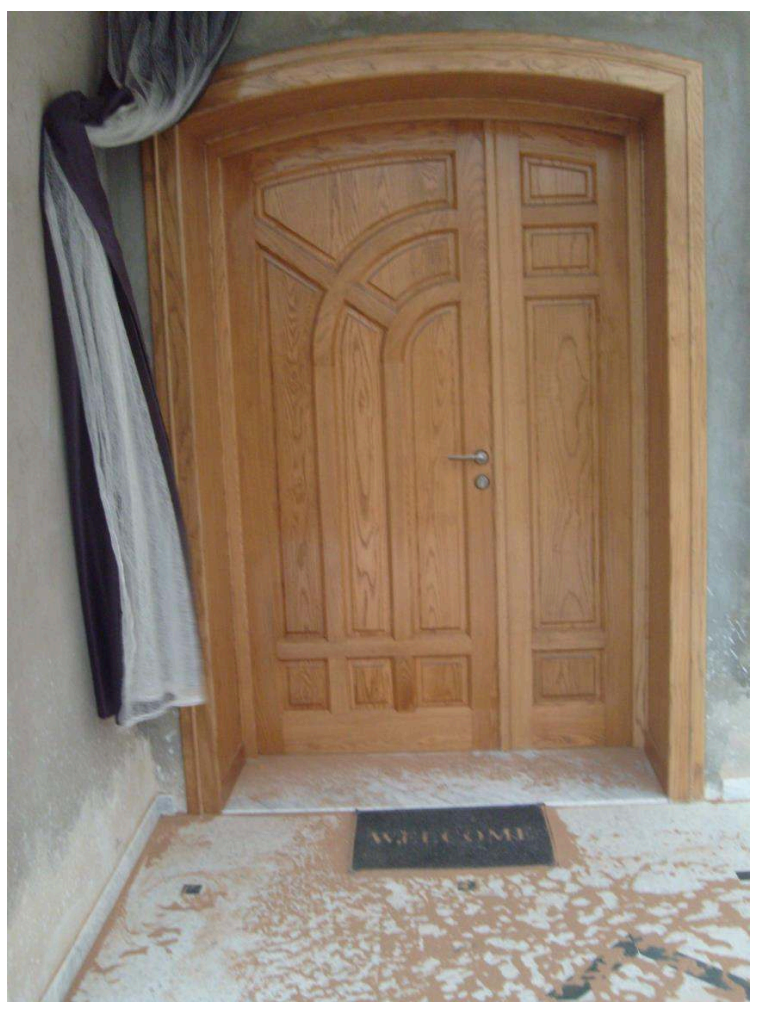

Source : Clichés Jarraya M., 2015

45 Nos enquêtes d'avril 2015 dans les CSB montrent que $25 \%$ des personnes asthmatiques interrogées (l'effectif total est de 114 cas) attribuent la réactivation de leurs allergies respiratoires aux poussières liées au vent de sable. Cependant, ce pourcentage augmente notablement à $48,5 \%$ (l'effectif total est de 73 cas) en octobre 2015 lorsque l'effet des poussières s'est associé aux caractéristiques de la chaleur élevée qui accompagne le Sirocco. Bien que ce type de temps à Sirocco (caractérisé par une forte chaleur et une chute de l'humidité relative de l'air) soit plus fréquent en été (Hénia et Mougou, 1997), il peut survenir également durant le reste de l'année, en particulier au printemps et en automne (Dahech et al., 2007), c'est-à-dire les deux saisons qui connaissent une fréquence plus élevée du vent de sable. Cette part des personnes asthmatiques attribuant la réactivation de leurs allergies respiratoires aux poussières liées au vent de sable fait l'objet d'une variation spatiale entre les CSB de Markez Kammoun et Sakiet-Ezzit ( $100 \%$ ) et les CSB de Ben Halima et Bouassida (0\%) (figure 14). En pénétrant profondément dans les voies respiratoires, les particules de poussières provoquent une réaction violente du système immunitaire sous formes de toux et dyspnée. Si la chaleur élevée représente un véritable risque pour les personnes atteintes par les maladies respiratoires (Jarraya et al., 2006), la présence de poussière dans l'air chaud constitue un danger supplémentaire qui peut alourdir la morbidité. L'association du vent de sable au Sirocco (des températures qui dépassent parfois $40^{\circ} \mathrm{C}$ ) qui souffle du secteur méridional, aggrave la situation déjà ardue des asthmatiques sensibles aux poussières. Une telle situation met leur vie en jeu. De ce fait, la consultation directe du service hospitalier d'urgence est fréquente pour faire face aux éventuelles difficultés respiratoires. A titre préventif, il ressort de nos entretiens que dans la plupart des cas, les asthmatiques sensibles aux poussières de sable (en 
particulier les personnes âgées) restent chez eux en cas de soufflement du vent de sable pour éviter toutes éventuelles complications.

Figure 14 : Variation des parts des poussières de sable dans les causes de consultations pour asthme selon les CSB en octobre 2015

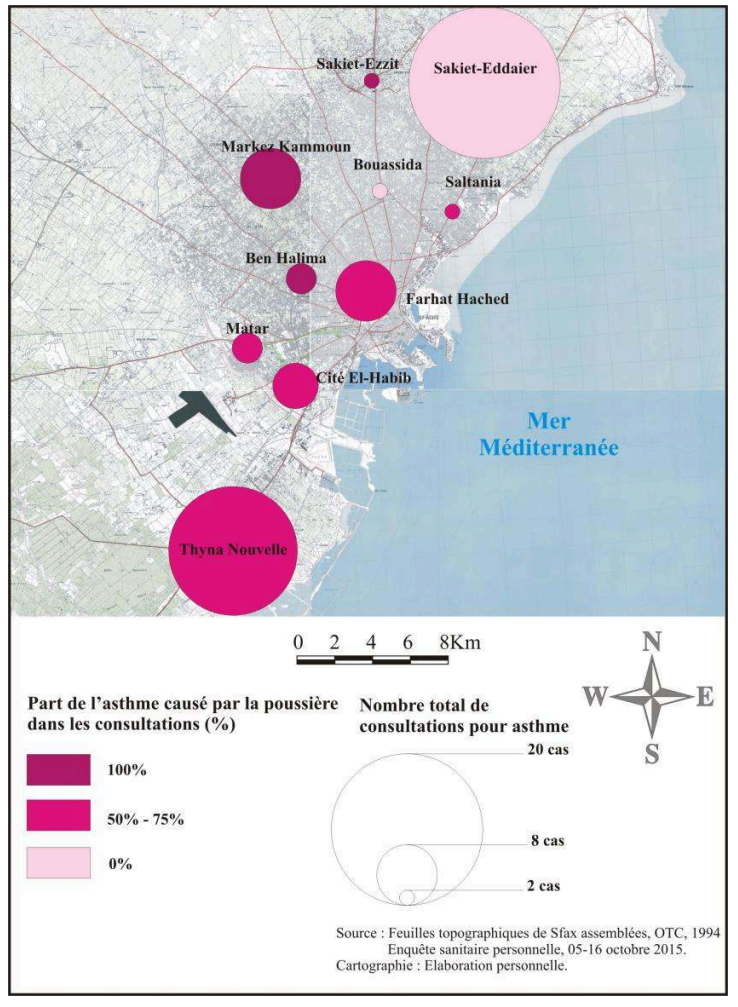

\section{L'effet d'une pollinisation intense}

Le pollen est le grain contenant les cellules reproductrices chez les plantes. La dispersion des grains de pollens des arbres en vue de la fécondation fait intervenir le vent comme vecteur essentiel (Gaussorgues, 2009).

Toutefois, pour que le pollen provoque une allergie, il faut qu'il soit allergisant, c'est-àdire il doit disposer des substances (protéines ou glycoprotéines) reconnues comme immunologiquement néfastes pour une personne donnée. Dans ce cas, l'allergie au pollens s'appelle pollinose ou rhume des foins (Laaidi et al., 1997).

Le printemps est la saison de floraison pour les espèces végétales. Certaines espèces comme l'olivier, qui prédomine dans le bassin méditerranéen, ont fait l'objet de plusieurs recherches portant sur les risques d'allergie qu'elles présentent pour la population (Pajaronet al., 1997 ; Gaussorgues, 2009). Certaines recherches ont attribué une part prépondérante au pollen de l'olivier dans l'explication des fortes prévalences des allergies respiratoires dans les pays méditerranéens (Charpin et Dutau, 1998; Demoly et al., 2005). L'olivier est une espèce prédominante dans l'arrière-pays de l'agglomération de Sfax. Ses pollens disposent un pouvoir allergisant important (Jarraya, 2009). Selon le Réseau National de Surveillance Aérobiologique (RNSA), l'olivier a un potentiel allergisant fort, c'est-à-dire que ses pollens présentent un véritable problème pour les personnes souffrant d'une telle allergie (www.pollens.fr). L'augmentation de la température et la diminution concomitante de l'humidité relative 
de l'air au printemps ainsi que la prédominance des situations aérologiques caractérisées par la fréquence du vent actif modéré constituent des conditions favorables pour le transport des pollens et sont à l'origine d'une forte concentration pollinique de l'air.

Notre enquête dans les CSB, en avril 2015, a permis de souligner la part prépondérante des agents allergènes végétaux dans les consultations pour asthme dans les CSB, ainsi que dans le traitement anti-allergique. Cette part des allergènes végétaux qui est de $84 \%$ de l'effectif total enquêté (114 cas) fait l'objet d'une variation spatiale entre les CSB. Elle varie de $100 \%$ dans les CSB de Ben Halima et Markez Kammoun à $60 \%$ dans le CSB de Saltania (figure 15). En outre, le taux des personnes qui déclarent le pollen d'olivier comme l'allergène végétal responsable de leur asthme est de $90 \%$ parmi les individus allergiques aux allergènes végétaux.

Figure 15 : Variation des parts des allergènes végétales dans les consultations pour asthme selon les CSB en avril 2015

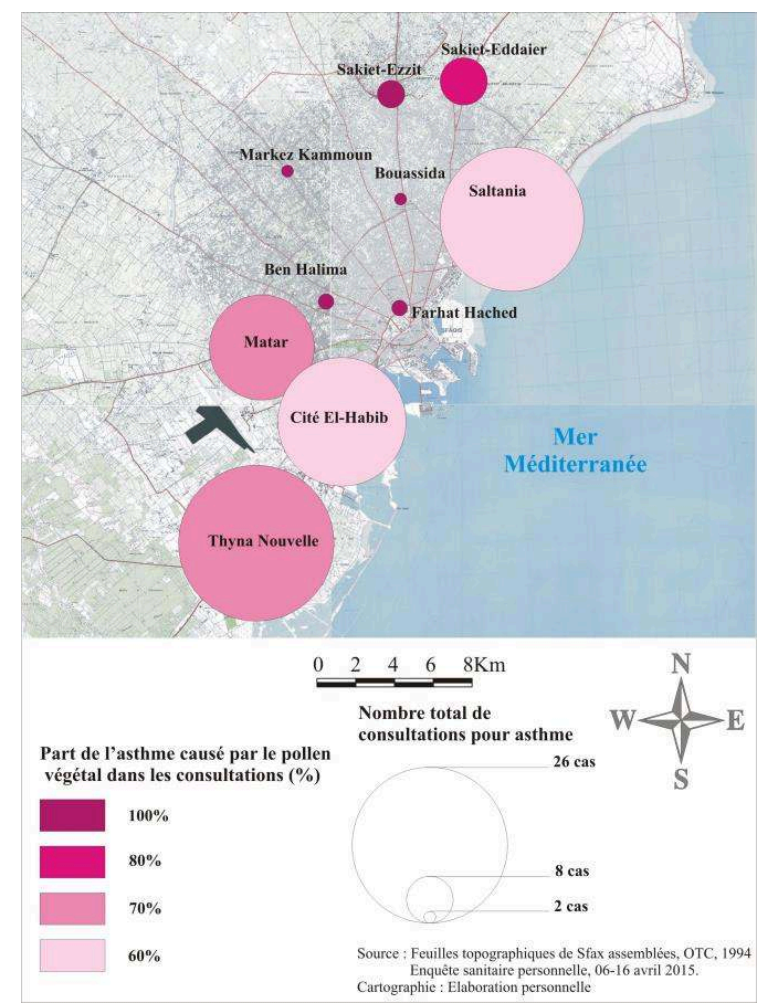

D'un autre côté, les pharmaciens publics (dans les CSB) et privés (quatre pharmacies dans la ville de Sfax) que nous avons interrogés ont enregistré une recrudescence des demandes de médicaments anti-allergiques. Ceux-ci se composent des antihistaminiques, des broncho-dilatateurs et des anti-asthmatiques. Des médicaments anti-histaminiques comme Allergamine, Allergica, Cetolergie, Fenadex, Telfast, Deslor... ainsi que d'autres broncho-dilatateurs comme Foradil, Bronchodual, Buto-Asma, Dilatrane, Seretide... sont largement servis par les pharmaciens au printemps pour soulager les atteintes respiratoires allergiques, en particulier l'asthme. Certains pharmaciens évaluent cet accroissement des demandes à $80 \%$ par rapport à l'hiver. La campagne environnante de la ville de Sfax est marquée par l'extension de « la forêt d'olivier ». Cela permet de suggérer de l'impact prévisible de ses pollens injectés dans 
l'air sur la santé respiratoire au cours de la saison de floraison. Compte tenu du rôle du vent dans la diffusion des pollens dans l'atmosphère de l'agglomération (figure 16), nous suggérons qu'à l'exception de la direction Est, qui est maritime, les autres directions soient des directions de vent à risque d'apport de pollens (Jarraya, 2009).

Figure 16 : L'olivier prédominant l'arrière pays de l'agglomération de Sfax est brassé par le vent

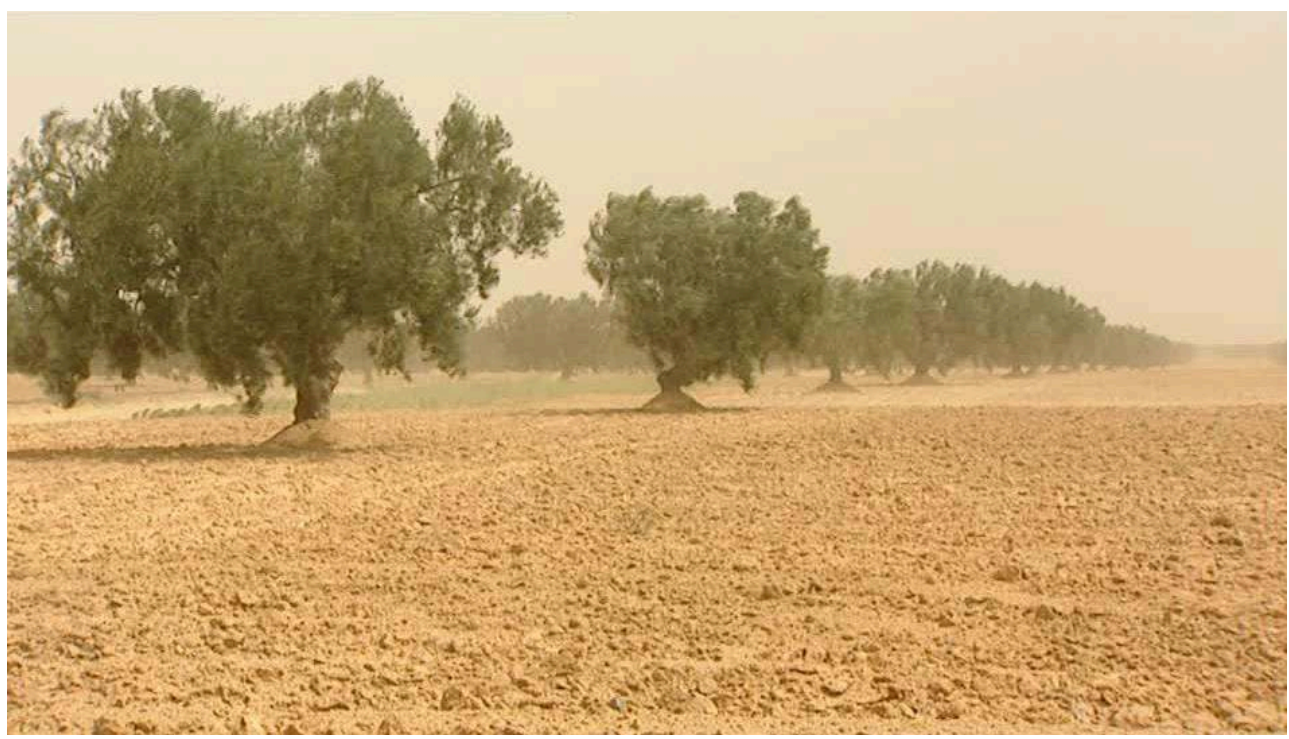

Source : http://footage.framepool.com/fr/bin/15851,culture+d \%27oliviers,sfax,tunisie/

La mise en cause des pollens de l'olivier dans la morbidité liée à l'asthme est expliquée par le fait que ceux-ci sont allergisants et de très petites tailles (de 20 à 60 micromètres en moyenne). Une telle taille leur permet d'atteindre facilement les voix respiratoires inférieures, en provoquant la toux, la respiration sifflante et l'asthme avec diminution du souffle. L'atteinte des voix respiratoires supérieures se matérialise par des rhinites avec irritation et picotements du nez, crises d'éternuement, écoulement souvent abondant et obstruction nasale.

Si les facteurs généraux d'ordre synoptique supposent que toute la population est exposée à l'éventuel risque lié aux poussières du vent de sable et aux pollens pendant la phase de floraison, les facteurs intrinsèques permettent de prendre en considération les spécificités socio-spatiales et environnementales de chaque délégation, afin de comprendre les disparités spatiales de la morbidité d'asthme. Par ailleurs, des mesures peuvent être entreprises, pour lutter contre cette multiplicité de sources de dégradation du bien-être et de santé respiratoire.

\section{Pour une réelle amélioration du bien-être dans le grand Sfax}

L'objectif de l'amélioration de la santé respiratoire implique une mobilisation multiacteurs permettant aux initiatives locales d'émerger. Deux types d'actions sont proposées pour lutter contre l'asthme dans le l'agglomération de Sfax : le premier type a pour vocation de lutter contre les facteurs environnementaux incriminés et d'agir sur 
les facteurs de risque et le second type se donne pour objectif la prévention de la maladie, en sensibilisant la population aux dangers de l'asthme.

\section{La lutte contre les facteurs environnementaux incriminés}

55 Compte tenu de l'aggravation de la santé des asthmatiques en particulier, la délocalisation de la SIAPE, qui constitue une source de pollution gazeuse, est fortement requise. Bien que la décision de son transfert à Skhira au Sud de Sfax, ait été prise depuis 2009, elle est encore loin de se concrétiser. Cette délocalisation s'inscrit dans l'effort de dépollution et d'aménagement du littoral de Sfax entrepris depuis deux décennies. Nous notons par exemple que la seconde unité de traitement de phosphate NPK datant de 1963 et qui a été installée dans la zone centrale de Sfax a été délocalisée en 1992. En outre, la décharge municipale de Thyna a été fermée depuis 2007 et délocalisée à $20 \mathrm{~km}$ au Sud-Ouest de la ville. C'est dans le même contexte qu'en 1990, les bassins de séchage des margines limitrophes à la SIAPE ont été fermés. Si ces initiatives montrent une volonté à réhabiliter la situation environnementale dans la ville de Sfax, la mobilisation de la société civile se maintient de plus en plus pour opérationnaliser la décision de délocalisation de la SIAPE, car les solutions provisoires (réduire la pollution par des filtres performants, renouvellement du matériel, nouveaux produits moins polluants...) n’ont pas allégé le niveau de pollution générée.

\section{Une sensibilisation sanitaire efficace}

Compte tenu de l'aspect multifactoriel de la morbidité liée à l'asthme, l'effort de la sensibilisation n'intéresse pas seulement les acteurs de la Santé Publique mais aussi les acteurs médiatiques. Si le secteur de la Santé de Base a introduit le programme national de l'Éducation sanitaire, qui adopte la communication comme outil principal pour tout effort préventif (DSSB, 2009), la médiatisation du problème de l'asthme en tant que risque pour la population urbaine contribue à instaurer une stratégie de lutte contre ses origines. Les différents outils médiatiques utilisés dans ce programme et qui se basent sur la communication de l'information préventive et curative (transmise à travers les affiches dans les différentes structures sanitaires réparties sur le territoire national, à travers les émissions télévisées et diffusées dans la radio et à travers les articles publiés dans les journaux et les sites internet) ont pour objectif de sensibiliser la population des dangers liés aux maladies et de mettre l'accent sur les lacunes au niveau régional (Jarraya, 2015).

$\mathrm{Du}$ point de vue opérationnel, l'éducation sanitaire repose sur plusieurs actes complémentaires. La production des outils éducatifs est fortement requise, car ils aident à la transmission des messages simples et compréhensibles par la population cible. En outre, cette sensibilisation cible aussi les causes de l'asthme, comme le traitement précoce des affections bronchiques avant qu'elles ne se compliquent. Compte tenu de l'effet aggravant du tabagisme, les divers moyens de sensibilisation vulgarisent le rôle du tabac dans la fragilisation de la santé des asthmatiques et de leurs proches. Une attention particulière est accordée aux enfants, puisqu'ils constituent des tabagiques passifs et fortement vulnérables aux fumées inhalées qui altèrent instantanément et à terme leur santé respiratoire. Si l'arrêt du tabac s'avère incertain 
pour les pères tabagiques et asthmatiques, des recommandations sont avancées pour réduire leur consommation de cigarettes.

De même, des mesures peuvent être suivies pour atténuer les effets néfastes des pollens. Si le pollen des oliviers est responsable de la recrudescence de l'asthme au printemps à Sfax, des campagnes de vaccination contre les allergies sont indispensables en hiver pour les personnes vulnérables, afin de limiter les complications de l'asthme. En outre, la forte relation qu'entretiennent les pollens avec les conditions météorologiques (essentiellement le vent) implique une coopération entre les services météorologiques et les spécialistes en allergologie, afin de prévenir à l'avance la population vulnérable d'une éventuelle concentration élevée du contenu pollinique de l'air. Cela nécessite la constitution d'une base de données sur le contenu pollinique de l'atmosphère mesurée dans les différents secteurs de l'agglomération de Sfax, afin d'étudier l'impact des pollens pendant la phase de floraison sur le bien-être et la santé des individus. Cela nécessite par exemple de préconiser aux personnes fortement sensibles (personnes âgées) de rester chez elles pendant ces épisodes de forte concentration pollinique dans l'air respiré.

Enfin, pour que les activités et les manifestations de sensibilisation soient efficaces, une couverture médiatique multimodale est nécessaire. Les chaînes de télévision nationales et les radios publiques émettent des séances animées par des spécialistes en pneumologie, allergologie et en pédiatrie et diffusent régulièrement des séquences de sensibilisation sur les dangers de l'asthme et sur les bonnes pratiques à suivre en cas de crises aigues.

Sans prétendre qu'elles soient exhaustives, ces mesures peuvent aider à lutter contre les causes de la maladie afin de baisser la morbidité liée à l'asthme dans l'agglomération de Sfax compte tenu les multiples répercussions sur le bien-être et la santé respiratoire.

\section{Discussion}

61 L'étude de la morbidité liée à l'asthme s'est basée sur des données épidémiologiques collectées par les CSB. Nous suggérons que l'agglomération de Sfax est suffisamment équipée en structures de soins primaires (27 CSB) pour permettre une couverture acceptable. Cependant, et en termes de représentativité des patients fréquentant les CSB par rapport à la totalité de la population de l'agglomération de Sfax, les études antérieures établies par les épidémiologues du Centre Hospitalo-Universitaire (CHU) Hédi Chaker de Sfax ont révélé que $60 \%$ de la population de Sfax se soignent dans les structures de soins publiques (Ben Jemaâ, 2004 ; Yaïch et al., 2010 ; Jarraya, 2016). Cela montre la position avancée qu'occupe le secteur de la Santé de Base en tant que première interface de la population avec le système sanitaire public qui intercepte les demandes primaires en soins. Dans le même contexte, les enquêtes établies ne peuvent pas être exhaustives, puisqu'elles sont limitées dans le temps et l'espace (dans dix CSB, pendant 10 jours). En l'absence d'une base de données fiable sur les asthmatiques, ce travail d'investigation sur les origines de l'asthme à Sfax reste insuffisant puisqu'il se base uniquement sur la disponibilité et le volontariat des médecins. C'est pourquoi la généralisation de ces résultats doit se faire avec prudence. de collecte des informations limités dans le temps et l'espace. Si certaines personnes 
jugent que les liens de causalité de l'asthme établis sont fondés sur les déclarations des patients, nous le justifions d'abord, par le fait que les individus perçoivent différemment l'effet de l'environnement sur leur état de santé. En outre, nous sommes conscients que la population souffre de différents types d'asthmes qui réagissent différemment envers les facteurs environnementaux. C'est pour cela que les enquêtes au niveau des asthmatiques sont fortement indispensables pour connaître la cause de cette maladie. Enfin, l'effet des éléments socio-comportementaux (comme le tabagisme et les conditions de logement) sur la morbidité liée à l'asthme ne peut être dévoilé qu'à travers des investigations au niveau des personnes asthmatiques enquêtées. Cependant, la référence à la biologie et les recherches établies sur des problématiques pareilles, est fortement requise afin d'appuyer nos hypothèses sur la causalité complexe de l'asthme dans l'agglomération de Sfax.

En termes de corps médical, les médecins pratiquant dans les CSB sont des généralistes. Cela pose un problème au niveau de l'aptitude de ceux-ci à diagnostiquer l'asthme, car la confirmation de la présence de cette maladie doit être basée sur des tests plus poussés. Ceux-ci doivent être effectués dans les structures hospitalières. De plus, le risque de la confusion avec d'autres atteintes du système respiratoire inférieur est fort possible, comme les Broncho-Pneumopathies Chroniques Obstructives (BPCO) qui présentent des symptômes similaires avec l'asthme. Il ressort de nos entretiens avec les médecins en poste dans les CSB que le diagnostic de l'asthme repose sur des signes bien précis (toux et respiration sifflante) qu'ils connaissent bien. Ils comptent beaucoup sur une symptomatologie spécifique, pour confirmer l'apparition de la maladie pour un nouvel patient et sur l'historique de la maladie chez les anciens patients. Les patients souffrant d'un asthme font l'objet d'un transfert quasi-immédiat vers les structures hospitalières pour des examens et des traitements spécialisés.

Notre présente approche souffre de l'absence d'informations sur le contenu pollinique de l'air, afin de mieux analyser le lien asthme-pollens au printemps. L'utilisation de ces données serait bénéfique pour mieux appréhender la question des allergies respiratoires à Sfax. Un tel problème sanitaire prédominant nécessite une attention particulière pour créer un dispositif rigoureux susceptible de mesurer les pollens et de mettre en place une base de données fiables pour toutes les éventuelles recherches. Cela est d'autant plus primordial que toute stratégie de lutte contre les allergies respiratoires nécessite le recours à des données précises concernant la production, la libération et la dissémination spatiale des pollens à Sfax.

65 Nous avons évoqué la pollution industrielle générée par la SIAPE comme facteur principal et explicatif des forts niveaux de morbidité liée à asthme dans le Sud et l'Ouest de l'agglomération, mais cela ne néglige pas l'effet d'autres sources de pollution atmosphérique comme le trafic routier qui représente un facteur important (Dahech et al., 2006). Sous l'effet de l'accroissement démographique, du grandissement de la taille de la ville, de la faiblesse du transport collectif et de l'utilisation fréquente par les individus des deux roues et de la voiture particulière dans leur déplacement à l'intérieur de l'agglomération, les volumes des polluants générés ne doivent pas être négligés.

Si nous avons évoqué dans notre présent travail l'effet du vent de sable et son apport en poussières dans l'atmosphère de l'agglomération pour expliquer le pic automnal de l'asthme, d'autres éléments de l'environnement peuvent aussi déclencher cette maladie. La chaleur et l'humidité élevées qui caractérisent l'ambiance automnale 
favorisent l'activité des moisissures. Celles-ci sont des champignons qui libèrent des substances provoquant l'irritation des voies aériennes. Cela aboutit à la réapparition de l'asthme extrinsèque en automne (Jarraya et Beltrando, 2016).

67 En partant des causalités complexes de l'asthme, nous notons que les allergies alimentaires et aux animaux peuvent les provoquer (Juchet et Broué-Chabbert, 2009 ; de Blay et Krieger, 1997). Même si nous nous sommes intéressés uniquement aux facteurs environnementaux favorisant l'asthme, nous sommes conscients de l'importance de ces allergies, qui sont considérées comme des déterminants comportementaux de l'asthme liés au régime alimentaire pratiqué. Dans ce cas, les effets peuvent être évitables si les individus sensibles contrôlent leur alimentation et s'éloignent des animaux.

\section{Conclusion}

Même si la part de l'asthme dans l'ensemble des maladies chroniques enregistrées par le secteur de la Santé de Base dans l'agglomération de Sfax est faible, cette maladie se distingue par ses effets négatifs sur le bien-être des individus. Entre asthme intrinsèque et extrinsèque, la morbidité liée à l'asthme exprime une variation mensuelle qui s'explique par une multitude de facteurs. Les disparités spatiales de la morbidité liée à l'asthme reflètent des contextes socio-environnementaux différents. Des déterminants intrinsèques influencent sensiblement les consultations pour asthme enregistrées par les CSB dans chaque délégation. D'autres facteurs de type général exposent l'agglomération de Sfax à des phénomènes d'ampleur spatiale vaste mais d'un effet plus ou moins temporaire. Les conditions d'habitat et de vie d'une manière générale ainsi que le comportement tabagique constituent deux déterminants primordiaux d'une morbidité liée à l'asthme élevée. Ces deux facteurs expliquent plutôt les types d'asthme intrinsèques dont les symptômes persistent durant toute l'année. La proximité des secteurs résidentiels densément peuplés aux industries polluantes contribue à une dégradation permanente de l'air et donc à une vulnérabilité accrue des asthmatiques résidant dans les quartiers environnants. À cela s'ajoutent les effets des vents de sable et des pollens qui rehaussent temporairement la morbidité liée à l'asthme de type extrinsèque en ciblant les asthmatiques saisonniers.

En tant que pôle économique principal à l'échelle nationale, le rythme de la dégradation environnementale s'accélère à Sfax face à une négligence de la part des autorités publiques, qui n'ont pas concrétisé jusqu'à aujourd'hui la décision de la délocalisation de la SIAPE et de l'atténuation de la pollution. Abstraction faite des enjeux politiques qui retardent cette mesure, il y a lieu maintenant d'améliorer l'encadrement sanitaire des personnes vulnérables et de les sensibiliser, afin de prévenir l'apparition de l'asthme et de lutter contre ses causes. 


\section{BIBLIOGRAPHIE}

Adriel Gudiel H., Jorge Gudiel H., Lissié Tincopa A., Dutau G., Rancé F., 2009 : Etudes des sensibilisations aux allergènes chez les enfants asthmatiques âgés de plus de trois ans et habitant dans la zone Nord de Lima (Pérou), Revue Française d'Allergologie, 49, p. 409-409.

Aissa I., Gharsalli H., Khattab A., Driss L., Ghedira H., 2010, Etat de contrôle de l'asthme en Tunisie. La Tunisie médicale, vol. 88, $\mathrm{n}^{\circ}$ 2, p. 97-101.

Aschan-Leygonie Ch., Baudet-Michel S., Mathian H., Sanders L., 2013, Les inégalités de santé respiratoire entre villes : une approche géographique. Environnement Risque Santé, vol. 12, $\mathrm{n}^{\circ} 2$, p. 118-128.

Azri C., Maalej A. et Medhioub K., 2000, Etude de la variabilité des constituants de l'aérosol dans la ville de Sfax (Tunisie). Pollution atmosphérique, $\mathrm{n}^{\circ}$ 165, p. 121-129.

Baïz N., Annessi-Maesano I., 2012, Is the Asthma Epidemic Still Ascending. Clin Chest Med, 33 (3), p. 419-429.

Burnett RT., Dales RE., Raizenne ME., 1994, Effects of low ambient levels of ozone and sulfates on the frequency of respiratory admissions to Ontario Hospitals. Environ Res, 6S, p. 172-194.

Ben Jemaâ I. 2004, Indicateurs hospitaliers de l'activité de l'EPS Hédi Chaker en 2002, Thèse de doctorat, Université de Sfax, Faculté de Médecine.

Besancenot J-P., 2001 : Climat et santé, PUF, Paris.

Busse W., 1988, The contribution of respiratory infection to the pathogenesis of airway hyper reactivity. Chest, 93, p. 1076-1082.

Casset A., Purohit A., de Blay F., 2008, Devenir des allergènes dans l'arbre respiratoire. Revue Française d'Allergologie et d'Immunologie Clinique, 48, p. 147-149.

Charpin D., Dutau H., 1998, Prévalence de l'asthme et de la rhinite dans les pays du Nord de la Méditerranée. Revue Française d'Allergologie et d'Immunologie Clinique, nº 38, p. 150-153.

Chaumel M., La Branche S., 2008, Inégalités écologiques : vers quelle définition ? Espace Population Sociétés, $\mathrm{n}^{\circ}$ 1, p. 101-110.

Choura M., 2008, Evaluation environnementale de l'activité de transformation de phosphate dans la zone côtière Sud du Grand Sfax : perspectives d'aménagement et de réhabilitation. Projet SMAP III - Tunisie (2006-2008), Rapport provisoire.

Corburn J., Osleeb J., Porter M., 2006, Urban asthma and the neighbourhood environment in New York City. Health, 12, p. 167-179.

Congdon P., Shouls S., Curtis S., 1997, A multi-level perspective on small area health and mortality: a case study of England and Wales. Int J Popul Geogr, 3, p. 243-263.

Cooper RS., 2001, Social inequality, ethnicity and cardiovascular disease. Int J Epidemiol, 30, S48. Coude-Gaussen G., 1990, Les régions sources de poussières au Sahara. Sécheresse, $\mathrm{n}^{\circ}$ 2, p. 134-141. Cummins S., Curtis S., Diez-Roux AV., Macintyre S., 2007, Unders-tanding and representing place in health research : a relational approach. Soc Sci Med, 65, p. 1825-1838.

Dahech S., Beltrando G. et Quenol H., 2006, Brise de mer et pollution atmosphérique à Sfax (Tunisie). Pollution atmosphérique, $\mathrm{n}^{\circ}$ 190, p. 211-223. 
Dahech S., 2007, Le vent à Sfax (Tunisie), impacts sur le climat et la pollution atmosphérique. Thèse de doctorat de l'Université Paris VII.

Dahech S., Beltrando G., Daoud A., 2007, Le sirocco et son impact sur l'agriculture et la qualité de l'air dans la région de Sfax. Sécheresse , 18 (3), p. 177-184.

Dahech S., Daoud A., Beltrando G., 2011, Les inégalités spatiales de la qualité de l'air dans l'agglomération de Sfax et ses environs : Le cas Des températures, de la brise, du CO et SO. Cybergeo : European Journal of Geography [En ligne], consulté le 30 juillet 2017. URL : http:// cybergeo.revues.org/24701

Dahech S., Beltrando G., 2012, Le vent de sable dans le Sud tunisien : conséquences pour l'Homme à Sfax. La Météorologie, $\mathrm{n}^{\circ} 79, \mathrm{p}$. 40-50.

de Blay F., Krieger P., 1997, Les allergies aux principaux mammifères domestiques et leur traitement. Revue Française d'Allergologie et d'Immunologie Clinique, $n^{\circ} 37$ (1), p. 56-64.

Dahech S., Bouaziz R., 2012, Risque de pollution atmosphérique sur la côte sud de l'agglomération de Sfax (Tunisie) : sensibilité de la population vulnérable. Actes de $25^{\text {ème }}$ Colloque de l'Association Internationale de Climatologie, Grenoble, p. 189-194.

Deger L., 2009, Facteurs environnementaux associés à un asthme mal maîtrisé chez les enfants montréalais. Mémoire de maîtrise des Sciences, Université de Montréal, Faculté de Médecine.

Demoly P., Godard P., Bousquet J., 2005, Une synthèse sur l'épidémiologie de l'asthme. Revue Française d'Allergologie et d'Immunologie Clinique, 45, p. 464-475.

Devouassoux G., 2003 : Allergies respiratoires chez l'enfant et chez l'adulte. Corpus médical, Faculté de médecine de Grenoble.

Diez-Roux A., Mair C., 2010, Neighborhood and health. Ann N Y Acad Sci, 1186, p. 125-45.

DSSB (Direction de Soins de Santé de Base), 2009, Le rapport annuel de 2009. Ministère de la Santé Publique, Tunis.

Emelianoff C., Theys J., 2000, Quand inégalités sociales et inégalités écologiques se cumulent, Annexe 2, in Développement durable, Villes et Territoires. Décloisonner pour anticiper les ruptures, J. Theys (dir.), Notes du Centre de Prospective et de Veille Scientifique, $\mathrm{n}^{\circ} 13$, Paris, 71-74.

Gaussorgues R., 2009, L'olivier et son pollen dans le bassin méditerranéen. Un risque allergique? Revue Française d'Allergologie et d'Immunologie Clinique, 49, S2-S6.

Hénia L., Mougou R., 1997, Contribution à l'étude des phénomènes atmosphériques à risque en Tunisie : Le cas du sirocco. Publication de l'Association Internationale de Climatologie, $\mathrm{n}^{\circ} 10$, p. 84-90.

Holifield R., 2000, Defining Environmental Justice and Environmental Racism. Urban Geography, vol. $22, \mathrm{n}^{\circ} 1$, p. $78-90$.

Jarraya M., Beltrando G., Ben Jemaa M., Damak J., 2006, Sirocco et pathologies respiratoires dans le secteur public de la Santé à Sfax (Tunisie). Actes du XIX ème colloque de l'Association Internationale de Climatologie, Épernay 06-09 septembre, p. 334-339.

Jarraya M., 2009, Biométéorologie de la morbidité respiratoire dans le secteur public de la santé à Sfax (Tunisie). Thèse de doctorat, Université Paris Diderot (Paris VII). 
Jarraya M., Beltrando G., 2013, Les services de soins hospitaliers publics dans l'agglomération de Sfax : spécificités fonctionnelles et pouvoir spatial. EchoGéo, $21 \mathrm{p}$; mis en ligne le 19 décembre 2013. URL : http://echogeo.revues.org/13632 ; DOI : 10.4000/echogeo.13632.

Jarraya M., 2015, Stratégie de communication sanitaire dans un territoire : cas de lutte contre la tuberculose dans le gouvernorat de Sfax en Tunisie. In Communication publique et développement territorial : Enjeux d'une valorisation et défis pour les acteurs, Merah A., Meyer V. (dir.), 129-150.

Jarraya M., Beltrando G., 2016, Poussés d'asthmes à Sfax (Tunisie) : aspects multiples de vulnérabilité accrue par le contexte climatique. Actes du XXIX ${ }^{\text {ème }}$ Colloque de l'Association Internationale de Climatologie, Besançon-Lausanne 06-09 juillet, p. 137-142.

Jarraya M. 2016, Bioclimatologie des infections cutanées mycosiques à Sfax (Centre-Est de la Tunisie). EchoGéo [En ligne], 38 | 2016, URL : http://echogeo.revues.org/14764 ; DOI : 10.4000/ echogeo.14764

Joobeur S., Cheikh Mhamed S., Ben Saad A., Mribah H., Dekhi A., Rouatbi N., El Kamel A., 2015, L'asthme allergique au centre tunisien. Pan Africain Medical Journal, http://www.panafricanmed-journal.com/content/article/20/133/full

Juchet A., Broué-Chabbert A., 2009, L'allergie alimentaire : ses liens avec l'asthme. Table ronde "Alimentation et pathologies respiratoires", Elsevier Masson, 16, p. 606-608.

Kallel M., 2008, Gestion intégrée de la zone côtière Sud de Sfax. Communication présentée à Tripoli, 29-31 janvier 2008.

Khot A., Burn R., Evans N., Lenney C., Lenney W., 1984, Seasonal variation and time tends in childhood asthma in England and Wales 1975-1981. Br Med J, 289, p. 235-237.

Laaidi K., Laaidi M., Besancenot J-P., 1997, Pollens, pollinoses et météorologie. La Météorologie, $8^{\text {ème }}$ série, $\mathrm{n}^{\circ} 20$, p. 41-56.

Laiidi K., 1999, Eco-épidémiologie des pollinoses en Hautes-Bourgognes. Thèse de doctorat, Université de Bourgogne, Faculté de Médecine.

Lean G., 2004, Sécurité environnementale. Our Planet , vol. 15, nº 4, PNUE, p. 16-17.

Lieutier-Colas F., Purohit A., Pauli G., de Blay F., 2003, Importance de la taille des particules dans la réponse bronchique à l'allergène, 43, p. 36-39.

Ministère de l'emploi et de la solidarité, 2001 : Programme d'action, de prévention et de prise en charge de l'asthme 2002-2005, Paris.

Obrist B., 2006, Risque et vulnérabilité dans la recherché en santé urbaine, Vertigo, Hors série, $\mathrm{n}^{\circ}$ 3, décembre 2006, mis en ligne le 01 décembre 2006, consulté le 20 mai 2014. URL : http:// vertigo.revues.org/1483; DOI : 10.4000/vertigo.1483.

Paddeu F., 2013, De la santé environnementale à la justice environnementale : l'enjeu de l'asthme infantile dans le South Bronx (New York), Développement durable et territoires [En ligne], vol. 4, $\mathrm{n}^{\circ} 2$ | Juillet 2013, mis en ligne le 16 juillet 2013, consulté le 13 avril 2014. URL : http:// developpementdurable.revues.org/9779 ; DOI:10.4000/developpementdurable.9779.

Pajarón MJ., Vila L., Prieto I., Resano A., Sanz ML., Oehling AK., 1997, Cross-reactivity of Olea europaea with other Oleaceae species in allergic rhinitis and bronchial asthma. Allergy, 52, p. 829-835.

Rican S., Jougla E., Salem G., 2003, Inégalités socio-spatiales de mortalité en France. Bulletin Epidemiologique Hebdomadaire, 30-31, p. 142-145. 
Riva M., Curtis S., Gauvin L., Fagg J., 2009, Unravelling the extent of inequalities in health across urban and rural areas : evidence from a national sample in England. Soc Sci Med, 68, p. 654-663.

Salem G., Rican S., Jougla E., 2006, Atlas de la santé en France, vol 1 : les causes de décès. Paris : John Libbey Eurotext.

Thibaudon M., Outteryck R., Lachasse C., 2005, Bioclimatologie et allergie, Revue Française d'Allergologie et d'Immunologie Clinique, 45, p. 447-455.

Thomson N., Chaudhuri R., Heaney L., Bucknall Ch., Niven R., Brightling Ch., Menzies-Gow A., Mansur A., McSharry Ch., 2013, Clinical outcomes and inflammatory biomarkers in current smokers and exsmokers with severe asthma. The Journal of Allergy and Clinical Immunology, Vol. 131, issue 4, p.1008-1016.

Turki, I., 2008, Etats de santé des tunisiens en 2030, Mémoire de Mastère, Université virtuelle de Tunis.

White K., Borrell LN., 2011, Racial/ethnic residential segregation: framing the context of health risk and health disparities. Health Place, 17, p. 438-48.

Yaïch S., Jdidi J., karray A., Fki H., Kassis M., Damak J., 2010, La Morbidité Hospitalière des Adolescents à Sfax - Tunisie, La Tunisie Médicale, Vol. 88 ( $\mathrm{n}^{\circ}$ 06), p. 399-403.

http://www.pollens.fr/le-reseau/les-pollens.php, Site de Réseau National de Surveillance Aérobiologique (RNSA). Les pollens / principaux pollens allergisants (consulté le 10/10/2017)

\section{ANNEXES}

\section{Annexe}

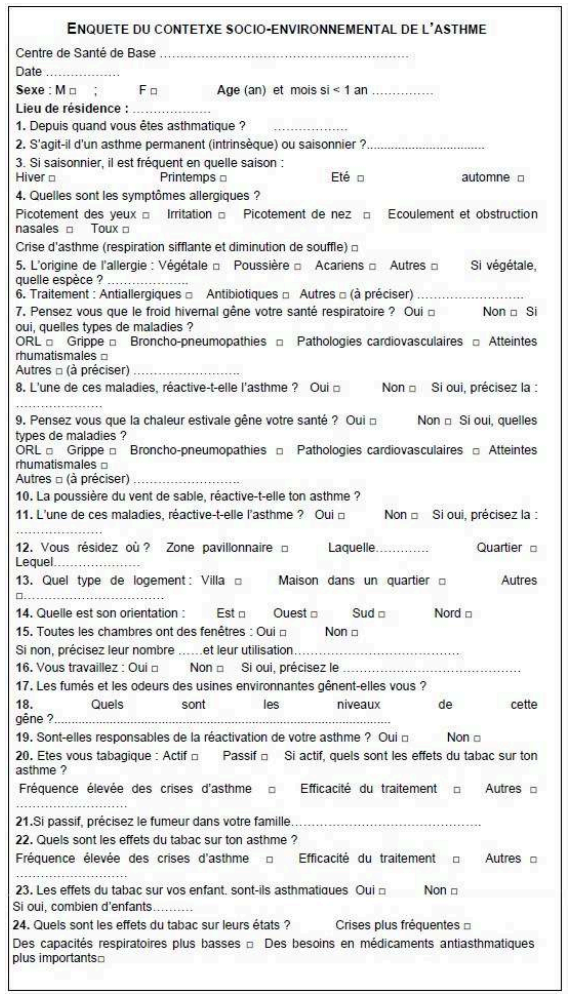




\section{RÉSUMÉS}

Notre travail traite de la relation santé-contexte socio-environnemental à travers l'analyse des disparités sanitaires de la morbidité liée à l'asthme dans l'agglomération de Sfax. La morbidité liée à l'asthme est exprimée à partir de l'effectif des consultations enregistrées dans les Centres de Santé de Base (CSB) sur la période 2009-2016. Si le diagnostic de la situation épidémiologique montre certaines caractéristiques liées à la variation selon les saisons et les classes d'âge, c'est la spatialisation des consultations pour asthme enregistrées par les CSB qui suscite l'intégration de multiples déterminants socio-environnementaux intrinsèques et généraux. Les facteurs intrinsèques, qui sont liés au contexte de vie et du comportement des individus, justifient la variation spatiale de la morbidité liée à l'asthme, alors que les facteurs dits généraux s'imposent pour expliquer la prédominance de l'asthme allergique extrinsèque. Compte tenu de cette combinaison complexe des déterminants de la santé des asthmatiques dans l'agglomération de Sfax, plusieurs mesures peuvent être suivies afin d'améliorer le bien-être et la santé respiratoire d'une population fortement « pénalisée » sur le plan environnemental.

This work deals with the relationship between health and socio-environmental context through the analysis of health disparities in asthma-related morbidity in the sfax agglomeration. This analysis is based on data obtained from the Basic Health Sector, which is the first line of recourse offering primary care services to the population in the various delegations in Sfax. The asthmarelated morbidity is expressed by the number of consultations recorded in the Basic Health Centers (BHC) over the period 2009-2016.

The diagnosis of the epidemiological situation of asthma shows the permanence of the consultations for asthma in the dispensaries of the agglomeration throughout the year. The monthly consultations recorded by the Health Sector Database show two peaks in April and October. This seasonal pattern is influenced by the factors of the environment and specially the climate. From the point of view of individual's vulnerability, the analysis of the epidemiological data by age shows a notable predominance of the adult category. However, this does not obscure the risk of asthma for other age groups, especially for children. The spatialization of asthma consultations recorded by BHC leads to the integration of multiple intrinsic and general socioenvironmental determinants. Factors related to the context of living (accommodation conditions) and behavior (smoking) of individuals as well as others specifying some sectors of the city than others (industrial air pollution) justify the spatial variation of asthma-related morbidity. Other so-called general factors spare people from their effects, such as sand-wind and pollen, and explain the spring and autumnal peaks of asthma-related morbidity in Sfax. These factors are necessary to lead to a predominance of extrinsic allergic asthma. Surveys carried out in BHC in April and October 2015 highlight some specificities of the asthma-related morbidity and its origins in the agglomeration of Sfax. The sand-wind, a fairly frequent phenomenon in sfax, alters the well-being of individuals and affects their respiratory health by reactivating asthma because of the very fine dust that can penetrate deeply into the lungs. The olive tree is the main species that causes respiratory allergies in Sfax as a plant pollen with a significant allergenic potential and a predominant asthma-related morbidity. Its effects become crucial for the vulnerable population and supported by a moderate wind and an ambient temperature which both favor the dissemination of the pollen.

Given this complex combination of determinants of asthma health in the agglomeration of Sfax, several measures can be taken to improve the well-being and respiratory health of an environmentally severely "penalized" population. Health awareness is a cornerstone of all strategies to fight respiratory allergies, which is at the origin of huge public health spending. However, the rehabilitation of respiratory health, which is severely impaired by multiple sources of nuisance, is based on the establishment of a healthy environment. This cannot be achieved 
without a genuine struggle against air pollution caused by the industrial units located in the south of the city, and in particular the Industrial Company of Phosphoric Acid and Fertilizer. The risks of asthma-related factors, particularly those related to the behavior of individuals such as smoking, can be mitigated by changing personal habits and following good practice leading ultimately to a significant reduction in the morbidity.

\section{INDEX}

Index géographique : Tunisie, Sfax

Mots-clés : disparités sanitaires, consultations médicales, asthme, contexte socioenvironnemental

Keywords : health disparities, medical consultations, asthma, social-environmental context, agglomeration of Sfax

\section{AUTEUR}

\section{MOUNIR JARRAYA}

Enseignant chercheur au laboratoire syfacte, Université de Sfax, Tunisie 